\title{
Complex dynamics of an impulsive chemostat model
}

\author{
Jin Yang*, Yuanshun Tan \\ College of Mathematics and Statistics, Chongqing Jiaotong University, \\ Chongqing, 400074,P.R.China, \\ *seehom@126.com \\ Robert A. Cheke \\ Natural Resources Institute, University of Greenwich at Medway, Central Avenue, \\ Chatham Maritime, Chatham, Kent, ME4 4TB, UK
}

\begin{abstract}
We propose a novel impulsive chemostat model with the substrate concentration as the basis for the implementation of control strategies, and then investigate the model's global dynamics. The exact domains of the impulsive and phase sets are discussed in light of phase portraits of the model and the Poincaré map and its complex properties were studied. Furthermore, the existence and stability of the microorganism eradication periodic solution are addressed, and analysis of a transcritical bifurcation reveals that an order-1 periodic solution is generated. In addition, the conditions for the global stability of an order-1 periodic solution are provided, and the existence of order $-k(k \geq 2)$ periodic solutions are studied. Moreover, the PRCC results and bifurcation analyses not only substantiate our results, but also indicate that the proposed system exists with complex dynamics. Finally, biological implications related to the theoretical results are discussed.
\end{abstract}

Keywords: Impulsive chemostat model; Poincaré map; Order- $k$ periodic solution; Transcritical bifurcation; Stability.

\section{Introduction}

The chemostat is an important laboratory apparatus which plays significant roles in ecological studies. It is used to investigate bacterial metabolism and population genetics because all conditions in it can be well controlled [Krishnaiah , 2007]. Many mathematical models have been proposed to study the culture of microorganisms in chemostats in relation to different reactions and control tactics [Leenheer \& Smith, 2003; Zhao \& Skogestad, 1997; Bailey \& Ollis, 1986; Butler \& Wolkowicz, 1985; Thierie, 2004]. Several papers have tried to improve the conditions of the chemostat so that species can coexist on the same substrate [Leenheer \& Smith , 2003; Kasperski \& Miskiewicz , 2008]. A Monod-type function has often been used to model the microorganisms' growth, which fits most biochemical reactions [Schugerl \& Bellgardt , 2000; Lobry et al., 1992; Sun \& Chen , 2007]. Meanwhile, the yield function is usually assumed to be a constant. However, the yield expression is not constant under certain conditions and Herbert's model and Pirt's model are suitable for quantification of maintenance energy in a microorganisms growth balance [Schugerl \& Bellgardt , 2000; Menkel \& Knights , 1995; Rehm \& Reed , 1981]. Base on these assumptions, the 
modified chemostat model can be described as:

$$
\left\{\begin{array}{l}
\frac{d S}{d t}=D\left(S_{F}-S\right)-(a+\exp (-b S)) \frac{\mu_{\max } S x}{S+K_{S}} \\
\frac{d x}{d t}=\frac{\mu_{\max } S x}{S+K_{S}}-D x
\end{array}\right.
$$

where $S$ and $x$ denote the concentrations of substrate and microorganism in a chemostat at time $t$, respectively. $D$ is the dilution rate, and $S_{F}>0$ is the concentration of the feed substrate, $b$ represents the sensitivity of the cell to the substrate when the cell is in a good environment, $a>1$ and $b>0$ are the biological constraints [Sun et al., 2010]. $\mu_{\max }$ is the maximal growth rate of the microorganism, $K_{S}$ is the saturation constant, the initial condition is denoted by $S\left(0^{+}\right)=S_{0}, x\left(0^{+}\right)=x_{0}$.

In a chemostat, the growth and reproduction of the microorganism population are affected by many key factors like temperature, $\mathrm{pH}$ and dissolved oxygen, which are usually controlled by human actions [Zhao \& Skogestad, 1997], with the microorganism population being determined by using a pump or an overflow system. The microorganism concentration, one of the two control criteria, is often used as a basis for the implementation of control strategies [Sun et al., 2011; Tian et al., 2010; Li et al., 2009; Yang et al., 2017; Guo \& Chen , 2009], and is detected by optoelectronic devices. If the microorganism concentration is lower than a critical threshold, then the pump is switched off or an overflow system is closed. Thereby, the yield increases because the substrate is consumed by the microorganism population. As the microorganism concentration increases and finally reaches an upper critical threshold, then the pump is switched on or the overflow system is opened, and a certain amount of substrate is allowed to flow in such that the microorganism concentration decreases and the secondary metabolites resulting from the microorganism production are also decreased [Li et al., 2009]. Such impulsive state feedback control can be described by an impulsive semi-dynamical system to provide a natural description for such control processes, and has been applied in many fields [Tang et al., 2015; Liu \& Chen , 2003; Zeng et al., 2006; Meng \& Chen , 2008; Huang et al., 2012]. Based on these ideas, a system (1) involving impulsive state feedback control can be described by:

$$
\left\{\begin{array}{l}
\frac{d S}{d t}=D\left(S_{F}-S\right)-(a+\exp (-b S)) \frac{\mu_{\max } S x}{S+K_{S}}, \\
\frac{d x}{d t}=\frac{\mu_{\max } S x}{S+K_{S}}-D x, \\
S\left(t^{+}\right)=(1-\delta) S(t), \\
x\left(t^{+}\right)=(1-\delta) x(t),
\end{array}\right\} \quad x=h,
$$

if the microorganism concentration $x$ reaches the threshold $h$, then the control strategy plays its role in decreasing the microorganism concentration and $0<\delta<1$ is the corresponding loss rate. System (2) has already been investigated by Sun and co-authors [Sun et al., 2011; Tian et al., 2010], who studied the existence and stability of order- 1 and order- 2 periodic solutions in some special cases, and they pointed out that there are no order- $k(k \geq 3)$ periodic solutions.

Notice that in system (2) the microorganism concentration is chosen as a controllable threshold. In the chemostat, there is a close relationship between the concentration of the substrate and the growth and reproduction of the microorganisms [Simkins \& Alexander , 1984, 1985]. Therefore, the concentration of the substrate (mainly the growth limiting factors of the microorganism) can also be chosen as a controllable threshold with which to apply a control strategy, which is different from system (2). Furthermore, the chemostat with the concentration of substrate as the monitored variable provides an important tool for research onmicrobial nutrition, growth, reproduction, metabolism and gene expression and regulation [Simkins \& Alexander , 1984, 1985]. The main feature is that the concentration of substrates are well-mixed so that environmental conditions are homogenous or uniform and microorganisms are randomly dispersed. If the dilution rate is low, then the concentration of the substrate would increase and finally reach a critical threshold value. Under this situation, a certain amount of the microorganism should be added into the chemostat, which not only guarantees the product yields and prevents a waste of substrate, but also allows the concentration of the substrate to be maintained within a reasonable range. If so, the microorganism 
concentration can be controlled below the critical value by means of controlling the concentration of substrate. Based on these ideas, model (2) can be extended by choosing the concentration of substrate as a controllable threshold. These modifications result in the following model

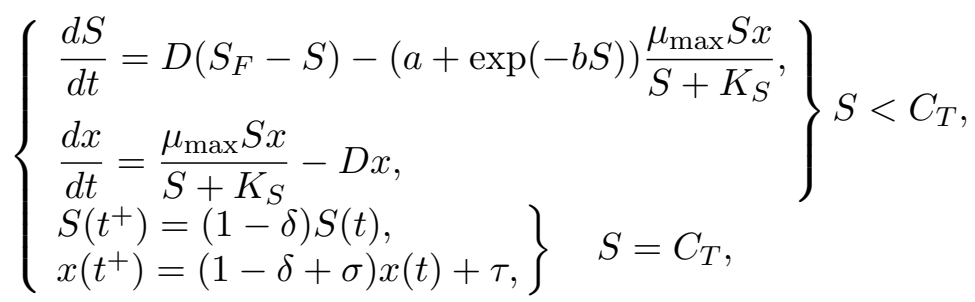

where $0 \leq \sigma<1$ and $\tau$ are the amount of the microorganism released, $\sigma>\delta$ stands for biological constraints and $C_{T}$ denotes the critical threshold of the substrate concentration. For convenience, it is assumed that the initial concentration of the substrate is less than the threshold $C_{T}$, otherwise, the initial values are taken after applications of control tactics. If the concentration of substrate reaches $C_{T}$ at time $t$, then a certain amount of the microorganism is added into the chemostat and the concentrations of substrate and microorganism are updated to $(1-\delta) C_{T}$ and $(1-\delta+\sigma) x(t)+\tau$, respectively.

The paper is arranged as follows. In section 2 , we introduce some useful definitions and lemmas about impulsive semi-dynamical systems, and the dynamics of system (3) without control are studied. In sections 3 and 4, the Poincaré map is first defined in relation to the exact domains of the phase sets. Thereafter, the important properties of the Poincaré map are investigated. Under certain conditions, the existence and stability of the microorganism eradication periodic solution are discussed, and the transcritical bifurcation is also addressed. Furthermore, the existence and stability of order $-k(k \geq 2)$ periodic solutions are also studied. In section 5, biological implications and conclusions are presented.

\section{Preliminaries and main properties of system (3)}

The planar autonomous dynamical systems with pulsed actions are usually defined by

$$
\left\{\begin{array}{l}
\frac{d S(t)}{d t}=P(S, x), \\
\frac{d x(t)}{d t}=Q(S, x), \\
S^{+}=S+\alpha(S, x), \\
x^{+}=x+\beta(S, x),
\end{array}\right\}(S, x) \in \mathcal{I},
$$

where $(S, x) \in R^{2}, P, Q, \alpha, \beta$ are continuous maps from $R^{2}$ to $R$, and $\mathcal{I} \subset R^{2}$ is denoted as the impulsive set. Let $S^{+}=S\left(t^{+}\right)$and $x^{+}=x\left(t^{+}\right)$. For any $z(S, x) \in \mathcal{I}$, the map $I: R^{2} \longrightarrow R^{2}$ can be defined

$$
z^{+}=I(z)=(S+\alpha(S, x), x+\beta(S, x))=\left(S^{+}, x^{+}\right) \in R^{2},
$$

$z^{+}$is the pulsed point of $z$.

Let $\mathcal{P}=I(\mathcal{I})$ be the phase set (i.e. for any $\left.z \in \mathcal{I}, I(z)=z^{+} \in \mathcal{P}\right)$, and $\mathcal{P} \cap \mathcal{I}=\varnothing$. System (4) can be defined as a planar impulsive semi-dynamical system.

The semi-dynamical system is usually denoted by $\left(X, \Pi, R_{+}\right)$or $(X, \Pi)$ [Simeonov \& Bainov , 1988; Bainov \& Simeonov , 1993], here $X$ is a metric space, $R_{+}$is set of non-negative reals. Denote $\Pi_{z}(t)=\Pi(z, t)$, for any $z \in X$, the map $\Pi_{z}: R \longrightarrow X$ is continuous such that $\Pi(z, 0)=z$ for all $z \in X$, and $\Pi(\Pi(z, t), s)=$ $\Pi(z, t+s)$ for all $z \in X$ and $t, s \in R_{+}$. Set $C^{+}(z)=\{\Pi(z, t) \mid t \in R\}$ represents the positive trajectory passing through $z$. For the impulsive set $\mathcal{I} \subset X$, let $\mathcal{I}^{+}(z)=C^{+}(z) \cap \mathcal{I}-\{z\}$ and $\mathcal{I}^{-}(z)=G(z) \cap \mathcal{I}-\{z\}$, where $G(z)=\cup\{G(z, t) \mid t \in R\}$ and $G(z, t)=\{w \in X \mid \Pi(w, t)=z\}$ is the attainable set of $z$ at $t \in R_{+}$. Finally, we set $\mathcal{I}(z)=\mathcal{I}^{+}(z) \cup \mathcal{I}^{-}(z)$. Now, we provide some useful Definitions and Lemmas about the semi-dynamical system [Ciesielski , 2004b,c; Kaul , 1990].

Definition 1. An impulsive semi-dynamical system $(X, \Pi ; \mathcal{I}, I)$ consists of a continuous semi-dynamical system $(X, \Pi)$, a nonempty closed subset $\mathcal{I}$ of $X$ and a continuous map $I: \mathcal{I} \longrightarrow X$, and further satisfies: No point $z \in X$ is a limit point of $\mathcal{I}(z) ;\{t \mid G(z, t) \cap \mathcal{I} \neq \varnothing\}$ is a closed subset of $R$. 
Denote the impulsive points of $\Pi_{z}$ by $\left\{z_{n}^{+}\right\}$, and then define a map $\ell$ from $X$ to the positive reals $R \cup\{\infty\}$ as: let $z \in X$, if $\mathcal{I}^{+}(z)=\varnothing$, then denote $\ell(z)=\infty$, otherwise $\mathcal{I}^{+}(z) \neq \varnothing$ and denote $\ell(z)=s$, where $\Pi(x, t) \notin \mathcal{I}$ for $0<t<s$ but $\Pi(z, s) \in \mathcal{I}$.

Definition 2. A trajectory $\Pi_{z}$ in $(X, \Pi, \mathcal{I}, I)$ is called and order $k$ period solution with period $T_{k}$ if there exist nonnegative integers $m \geq 0$ and $k \geq 1$ such that $k$ is the smallest integer for which $z_{m}^{+}=z_{m+k}^{+}$and $T_{k}=\sum_{i=m}^{m+k-1} \ell\left(z_{i}\right)=\sum_{i=m}^{m+k-1} s_{i}$.

Lemma 1. ([Simeonov \& Bainov, 1988; Bainov \& Simeonov, 1993]) The T-periodic solution $(x, y)=$ $(\xi(t), \eta(t))$ of system

$$
\left\{\begin{array}{l}
\frac{d S}{d t}=P(S, x), \quad \frac{d x}{d t}=Q(S, x), \quad \text { if } \quad \phi(S, x) \neq 0, \\
S^{+}=S+\alpha(S, x), \quad x^{+}=x+\beta(S, x), \quad \text { if } \quad \phi(S, x)=0,
\end{array}\right.
$$

is orbitally asymptotically stable if the Floquet multiplier $\mu_{2}$ satisfies the condition $\left|\mu_{2}\right|<1$, where

$$
\mu_{2}=\prod_{k=1}^{q} \Delta_{k} \exp \left(\int_{0}^{T}\left[\frac{\partial P}{\partial S}(\xi(t), \eta(t))+\frac{\partial Q}{\partial x}(\xi(t), \eta(t))\right] d t\right)
$$

with

$$
\Delta_{k}=\frac{P_{+}\left(\frac{\partial \beta}{\partial x} \frac{\partial \phi}{\partial S}-\frac{\partial \beta}{\partial S} \frac{\partial \phi}{\partial x}+\frac{\partial \phi}{\partial S}\right)+Q_{+}\left(\frac{\partial \alpha}{\partial S} \frac{\partial \phi}{\partial x}-\frac{\partial \alpha}{\partial x} \frac{\partial \phi}{\partial S}+\frac{\partial \phi}{\partial x}\right)}{P \frac{\partial \phi}{\partial S}+Q \frac{\partial \phi}{\partial x}},
$$

and $\phi$ is continuously differentiable about $S, x .(S, x) \notin \mathcal{I}$ is also denoted as $\phi(S, x) \neq 0$. $P, Q, \partial \alpha / \partial S, \partial \alpha / \partial x, \partial \beta / \partial S, \partial \beta / \partial x, \partial \phi / \partial S$ and $\partial \phi / \partial x$ are calculated at the point $\left(\xi\left(t_{k}\right), \eta\left(t_{k}\right)\right), P_{+}=$ $P\left(\xi\left(t_{k}^{+}\right), \eta\left(t_{k}^{+}\right)\right)$and $Q_{+}=Q\left(\xi\left(t_{k}^{+}\right), \eta\left(t_{k}^{+}\right)\right) . t_{k}(k, q \in N, N$ is non-negative integers) is the time of the $k$-th jump.

Lemma 2. ([Rasband, 1990]) $F: R \rightarrow R$ denotes a one-parameter family of $C^{2}$ map and satisfies (1) $F(0, \rho)=0$ for all $\rho$; (2) $\frac{\partial F}{\partial x}(0,0)=1$;

(3) $\frac{\partial^{2} F}{\partial x \partial \rho}(0,0)>0$; (4) $\frac{\partial^{2} F}{\partial x^{2}}(0,0)<0$, if $\rho$ is near zero, then map $F$ exists with two branches of the fixed points. The first branch is $x_{1}(\rho)=0$ for all $\rho$, while the second bifurcating branch $x_{2}(\rho)$ changes its value from negative to positive as $\rho$ increases through $\rho=0$ with $x_{2}(0)=0$. The fixed points of the first branch are stable if $\rho<0$ and unstable if $\rho>0$, while those of the bifurcating branches have the opposite stability.

In order to study the global dynamics of system (3), we first need to know the dynamical behaviour of system (1) in the first quadrant. For system (1), the two isoclines are denoted by $L_{1}$ and $L_{2}$, where

$$
l_{1}: S=\frac{D K_{S}}{\mu_{\max }-D}, \quad l_{2}: x=\frac{D\left(S_{F}-S\right)\left(S+K_{S}\right)}{\mu_{\max } S(a+\exp (-b S))} .
$$

There exists a boundary equilibrium $\left(S_{F}, 0\right)$ which is a saddle, and a unique interior positive equilibrium $E^{*}\left(S^{*}, x^{*}\right)$ provided $D<\mu_{\max }$ and $K_{S}<\left(\mu_{\max } / D-1\right) S_{F}$, where

$$
S^{*}=\frac{D K_{S}}{\mu_{\max }-D}, \quad x^{*}=\left(S_{F}-S^{*}\right)\left(a+\exp \left(-b S^{*}\right)\right)^{-1} .
$$

Unless otherwise specified we assume that $D<\mu_{\max }$ and $K_{S}<\left(\mu_{\max } / D-1\right) S_{F}$ always hold throughout the paper. The characteristic polynomial of the system (1) about $E^{*}$ is

$$
\lambda^{2}+p \lambda+q=0
$$

where

$$
\begin{gathered}
p=D\left(\frac{\left(S^{*}\right)^{2}+K_{S} S_{F}}{S^{*}\left(K_{S}+S^{*}\right)}-\frac{b \exp \left(-b S^{*}\right)\left(S_{F}-S^{*}\right)}{\left(a+\exp \left(-b S^{*}\right)\right)}\right), \\
q=\frac{\mu_{\max } K_{S} D\left(S_{F}-S^{*}\right)}{\left(K_{S}+S^{*}\right)^{2}}>0 .
\end{gathered}
$$


Denote

$$
\theta=\left[b \frac{S^{*}\left(S_{F}-S^{*}\right)\left(K_{S}+S^{*}\right)}{\left(S^{*}\right)^{2}+K_{S} S_{F}}-1\right] \exp \left(-b S^{*}\right),
$$

then $p>0$ provided $a>\theta$. Thus, if $a \geq \theta$, then $E^{*}$ is stable; if $a<\theta$, then $E^{*}$ becomes unstable. Further, we have the following results [Sun et al., 2011; Tian et al., 2010].

Lemma 3. If $a \geq \theta$, then $E^{*}$ is asymptotically stable; if $a<\theta$, then $E^{*}$ becomes unstable and there exists a unique stable limit cycle for system (1).

Proof. If $a<\theta$, then $E^{*}$ is unstable. Let the line

$$
L_{\text {out }}: x(t)+\frac{S(t)}{a+\exp -b S_{F}}-\frac{S_{F}}{a+\exp -b S_{F}}=0,
$$

thus, the derivative of the line $L_{\text {out }}$ along the solutions of system (1) can be obtained

$$
\frac{d L_{\text {out }}}{d t}=\frac{\mu_{\max } S\left(S_{F}-S\right)}{\left(a+\exp \left(-b S_{F}\right)\right)\left(K_{S}+S\right)}\left(1-\frac{a+\exp -b S}{a+\exp -b S_{F}}\right)<0 .
$$

Besides, the $S(t)$ axis, $x(t)$ axis, the line $S(t)=S_{F}$ and the line $L_{\text {out }}$ constitute the outer boundary of the Poincaré-Bendixson ring domain, and the inner boundary shrinks to the unstable $E^{*}$. According to the Bendixson Theorem, there exists a limit cycle for system (1).

\section{Poincaré map and order $-k$ periodic solution}

In order to investigate the existence of order $-k$ periodic solutions of system (3), we first need to define the Poincaré map in the phase sets. In this case, the existence of order- $k$ periodic solutions can be realized by studying the fixed points of the Poincaré map. Define another two lines as follows:

$$
l_{3}: S=(1-\delta) C_{T}, \quad l_{4}: S=C_{T} .
$$

It follows from the biological significance that the concentration of the feed substrate $S_{F}$ satisfies $0<C_{T}<S_{F}$. Substituting $S=C_{T}$ into the line $l_{2}$, we get the intersection point of $l_{2}$ and $l_{4}$, denoted as $I_{P}\left(C_{T}, x_{C}\right)$ with

$$
x_{C}=\frac{D\left(S_{F}-C_{T}\right)\left(C_{T}+K_{S}\right)}{\mu_{\max } C_{T}\left(a+\exp \left(-b C_{T}\right)\right)} .
$$

And the intersection point of $l_{2}$ and $l_{3}$ is denoted by $I_{P}^{1}\left((1-\delta) C_{T}, x_{\delta C}\right)$ with

$$
x_{\delta C}=\frac{D\left(S_{F}-(1-\delta) C_{T}\right)\left((1-\delta) C_{T}+K_{S}\right)}{\mu_{\max }(1-\delta) C_{T}\left(a+\exp \left(-b(1-\delta) C_{T}\right)\right)} .
$$

Now, the most important thing for us is to discuss the exact domains of impulsive sets and phase sets such that the domains of the Poincaré map can be determined. To realize this purpose, according to the locations between the threshold $C_{T}$ and the equilibrium $E^{*}$, there are two possible cases needed to be considered:

$$
\left(A_{1}\right) \quad C_{T}<S^{*} \quad \text { and } \quad\left(A_{2}\right) \quad C_{T} \geq S^{*} .
$$

For case $\left(A_{1}\right)$, the phase set and impulsive set which lies on the lines $l_{3}$ and $l_{4}$ are both located in the region of the left hand of $E^{*}$. It follows from the vector fields of the system (3) that any solution starting from $l_{3}$ may reach $l_{4}$ in finite time. Therefore, the impulsive set $\mathcal{I}$ of system (3) is defined by

$$
\mathcal{I}=\left\{(S, x) \in R^{2} \mid S=C_{T}, 0 \leq x \leq x_{C}\right\},
$$

further $\mathcal{I}$ is a closed subset of $R^{2}$. For the phase set, we need to define a continuous function $I$ such that

$$
I:\left(C_{T}, x\right) \in \mathcal{I} \longrightarrow\left(S^{+}, x^{+}\right)=\left((1-\delta) C_{T},(1-\delta+\sigma) x+\tau\right) \in R^{2} .
$$


Then the phase set $\mathcal{P}$ can be defined by

$$
\mathcal{P}=I(\mathcal{I})=\left\{\left(S^{+}, x^{+}\right) \in R^{2} \mid S^{+}=(1-\delta) C_{T}, x^{+} \in D_{0}\right\}
$$

with $D_{0}=[\tau,(1-\delta+\sigma) x+\tau]$. Based on the above definitions, system (3) can be defined by impulsive semi-dynamical system $(X, \Pi ; \mathcal{I}, I)$. Unless otherwise specified we assume that the initial point $\left(S_{0}^{+}, x_{0}^{+}\right) \in$ $\mathcal{P}$. Moreover, notice that the solution of system (3) initiating from $\left(S_{0}^{+}, x_{0}^{+}\right)$with $x_{0}^{+}>(1-\delta+\sigma) x_{C}+\tau$ experiences a single impulsive effect and then satisfies $x_{k}^{+} \leq(1-\delta+\sigma) x_{C}$ for all $k \geq 1$, thus the domain of the Poincaré map is defined as $D_{0}=[\tau,+\infty)$.

For case $\left(A_{2}\right), l_{4}$ is on the right hand of $E^{*}$, while $l_{3}$ is either on the left or right hand of $E^{*}$. It follows from Lemma 2.3 that $E^{*}$ of system (3) is either stable or unstable, and there exists a unique stable limit cycle $\Omega$ if $E^{*}$ is unstable under different conditions. It means that the solution starting from $\left((1-\delta) C_{T}, x_{0}^{+}\right)$with $x_{0}^{+}>0$ will experience either infinitely many pulses or no impulsive effect. For example, if $E^{*}$ is stable, then there exists a curve $\Gamma$ which is tangential to $l_{4}$ at point $I_{P}\left(C_{T}, x_{C}\right)$, and $\Gamma$ intersects $l_{2}$ at point $P\left(S_{P}, x_{P}\right)$ such that $\Gamma$ is tangential to $l_{2}$ at this point. If $(1-\delta) C_{T}<S_{P}$, then any curves starting from $\left((1-\delta) C_{T}, x_{0}^{+}\right)$with $x_{0}^{+} \in D_{0}$ experiences infinitely many pulses. If $(1-\delta) C_{T} \geq S_{P}$, then $\Gamma$ has two intersect points with $l_{3}$, two points are denoted by $P_{1}=\left((1-\delta) C_{T}, x_{\min }\right)$ and $P_{2}=\left((1-\delta) C_{T}, x_{\max }\right)$. It is easy to see that any solution starting from $\left(S_{0}^{+}, x_{0}^{+}\right)$with $x_{\min }<x_{0}^{+}<x_{\max }$ will never experience an impulsive effect and finally tend to the stable $E^{*}$. Therefore, the domains of impulsive and phase sets should be discussed in order to provide the domains for the Poincaré map, which will be addressed in the next section.

\subsection{Complex properties of Poincaré map for case $\left(A_{1}\right)$}

In order to construct the Poincaré map for the impulsive points, we need to define two sections as follows

$$
S_{0}^{+}=\left\{(S, x) \mid S=(1-\delta) C_{T}, x \geq 0\right\}, \quad S_{0}=\left\{(S, x) \mid S=C_{T}, x \geq 0\right\} .
$$

Without loss of generality, section $S_{0}^{+}$is used to define the Poincaré map. For any point $H_{k}^{+}\left((1-\delta) C_{T}, x_{k}^{+}\right) \in$ $S_{0}^{+}$, the solution starting from $H_{k}^{+}$meets section $S_{0}$ at point $H_{k+1}\left(C_{T}, x_{k+1}\right)$ in a finite time, and $x_{k+1}$ is a function of $y_{k}^{+}$denoted as $y_{k+1}=g\left(y_{k}^{+}\right)$. After a single impulsive effect, point $H_{k+1}$ maps to the point $H_{k+1}^{+}\left((1-\delta) C_{T}, x_{k+1}^{+}\right) \in S_{0}^{+}$, where $x_{k+1}^{+}=(1-\delta+\sigma) x_{k+1}+\tau$. Then the Poincaré map for the impulsive points of system (3) can be defined as

$$
x_{i+1}^{+}=(1-\delta+\sigma) g\left(x_{i}^{+}\right)+\tau \triangleq \Phi\left(x_{i}^{+}\right) .
$$

Since the Poincaré map has been defined, the explicit expression is unknown. For the existence of periodic solutions of system (3), we only need to study the fixed points of the Poincaré map. To achieve this purpose, in the following we will determine the explicit expression of the Poincaré map according to the phase portrait of model (3). Thus, let

$$
\begin{aligned}
& P(S(t), x(t))=D\left(S_{F}-S\right)-(a+\exp (-b S)) \frac{\mu_{\max } S x}{S+K_{S}}, \\
& Q(S(t), x(t))=\frac{\mu_{\max } S x}{S+K_{S}}-D x,
\end{aligned}
$$

then we have the following scalar differential equation in phase space

$$
\left\{\begin{array}{l}
\frac{d x}{d S}=\frac{\frac{\mu_{\max } S x}{S+K_{S}}-D x}{D\left(S_{F}-S\right)-(a+\exp (-b S)) \frac{\mu_{\max } S x}{S+K_{S}}} \doteq \omega(S, x), \\
x\left((1-\delta) C_{T}\right)=x_{0}^{+} .
\end{array}\right.
$$

For model (9), the following important region $\mathbb{D}$ is considered, where

$$
\mathbb{D}=\left\{(S, x) \mid S>0, x>0, x<\frac{D\left(S_{F}-S\right)\left(S+K_{S}\right)}{\mu_{\max } S(a+\exp (-b S))}\right\},
$$

and function $\omega(x, y)$ is continuously differentiable in $\mathbb{D}$. Denote $S_{0}^{+}=(1-\delta) C_{T}, x_{0}^{+} \doteq \zeta, \zeta \in \mathcal{P}$ with $\zeta<x_{\delta C}$, i.e., $\left(S_{0}^{+}, x_{0}^{+}\right) \in \mathbb{D}$, This notation yields

$$
x(S)=x\left(S ;(1-\delta) C_{T}, \zeta\right)=x(S, \zeta), \quad(1-\delta) C_{T} \leq S \leq C_{T},
$$


thus from model (9) we have

$$
x(S, \zeta)=\zeta+\int_{(1-\delta) C_{T}}^{S} \omega(S, x(S, \zeta)) d S .
$$

Therefore, in the region $\mathbb{D}$ the explicit expression of the Poincaré map $\Phi$ of system (3) takes the form

$$
\Phi(\zeta)=(1-\delta+\sigma) x\left(C_{T}, \zeta\right)+\tau .
$$

Since the explicit expression of the Poincaré map has been provided, the corresponding properties must be discussed before we investigate the periodic solutions of system (3).
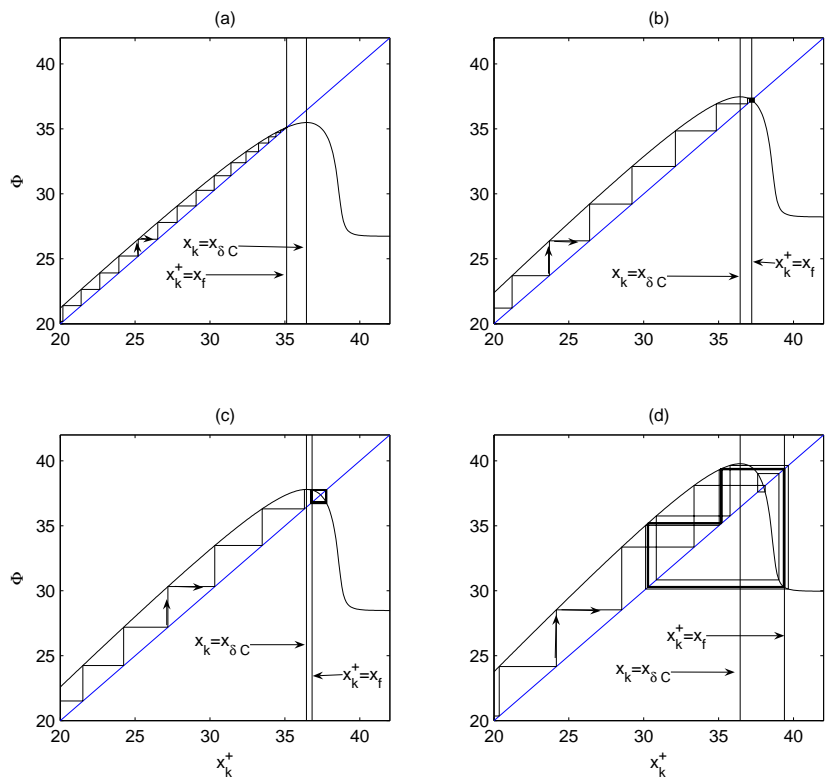

Fig. 1. The existence and locations of the fixed point $x_{f}$ with respect to the Poincaré map $\Phi$. The parameters are fixed as $D=0.15, S_{F}=6, a=0.1, b=5, \mu_{\max }=0.3, K_{S}=0.8, C_{T}=0.7, \delta=0.4$ and $\tau=0.2$. (a) $\sigma=0.47$; (b) $\sigma=0.53$; (c) $\sigma=0.54 ;$ (d) $\sigma=0.6$.

Theorem 1. For case $\left(A_{1}\right)$, the complex properties of the Poincaré map $\Phi$ are listed as follows (Fig.1):

(I) The domain and range of $\Phi$ are $[0,+\infty)$ and $\left[\tau, \Phi\left(x_{\delta C}\right)\right)=\left[\tau,(1-\delta+\sigma) x\left((1-\delta) C_{T}, x_{\delta C}\right)+\tau\right]$, respectively. It is increasing on $\left[0, x_{\delta C}\right]$ and decreasing on $\left[x_{\delta C},+\infty\right)$.

(II) $\Phi$ is continuously differentiable.

(III) $\Phi$ is concave on $\left[0, x_{\delta C}\right)$.

$(I V)$ there always exists a unique positive fixed point $x_{f}$ for $\Phi$.

$(V)$ there exists a horizontal asymptote $x=\tau$ for $\Phi$ when $x_{k}^{+} \rightarrow+\infty$.

Proof. (I) According to the properties of solutions in the phase plane of system (3) in the first quadrant, the domain of the Poincaré map $\Phi$ can be defined as $[0, \infty)$ for biological significance. For any $x_{i}^{+}, x_{j}^{+} \in\left[0, x_{\delta C}\right]$ with $x_{i}^{+}<x_{j}^{+}$, it follows from the Cauchy-Lipschitz theorem that we have $x_{i+1}<x_{j+1}$. After pulses, the inequality $(1-\delta+\sigma) x_{i+1}+\tau<(1-\delta+\sigma) x_{j+1}+\tau$ holds true, i.e., $\Phi\left(x_{i}^{+}\right)<\Phi\left(x_{j}^{+}\right)$. Thus, $\Phi$ is increasing on $\left[0, x_{\delta C}\right]$. If $x_{i}^{+}, x_{j}^{+} \in\left[x_{\delta C},+\infty\right)$ with $x_{i}^{+}<x_{j}^{+}$, from the qualitative theories of ordinary differential equations, it is found that at the left hand side of the isocline $l_{1}$ the variable $x(t)$ is decreasing, while $S(t)$ is first decreasing in the region above the isocline $l_{2}$ and then increasing in the region below the isocline $l_{2}$. It indicates that the solutions initiating from $\left((1-\delta+\sigma) C_{T}, x_{i}^{+}\right)$and $\left((1-\delta+\sigma) C_{T}, x_{j}^{+}\right)$will first reach 
$l_{3}$ at two points $\left((1-\delta+\sigma) C_{T}, x_{i}^{\prime}\right)$ and $\left((1-\delta+\sigma) C_{T}, x_{j}^{\prime}\right)$ with $x_{i}^{\prime}>x_{j}^{\prime}$, and then meet $l_{4}$ at $\left(C_{T}, x_{i+1}\right)$ and $\left(C_{T}, x_{j+1}\right)$ with $x_{i+1}>x_{j+1}$. After a single pulse, we have

$$
\Phi\left(x_{i}^{+}\right)=(1-\delta+\sigma) x_{i+1}+\tau>(1-\delta+\sigma) x_{j+1}+\tau=\Phi\left(x_{j}^{+}\right) .
$$

Therefore, $\Phi$ is decreasing on $\left[x_{\delta C},+\infty\right)$. In addition, the range of $\Phi$ is $\left[\tau,(1-\delta+\sigma) x\left((1-\delta) C_{T}, x_{\delta C}\right)+\tau\right]$.

(II) Since functions $P(S, x)$ and $Q(S, x)$ are both continuous and differentiable in the first quadrant, it follows from the Cauchy-Lipschitz theorem with parameters that $\Phi$ is continuous and differentiable. Moreover, functions $P(x, y)$ and $Q(x, y)$ are $C^{\infty}$, thus $\Phi$ is also $C^{\infty}$.

(III) It follows from equation (9) that

$$
\begin{aligned}
\frac{\partial \omega}{\partial x}= & \frac{\frac{\mu_{\max } S}{S+K_{S}}-D}{D\left(S_{F}-S\right)-(a+\exp (-b S)) \frac{\mu_{\max } S x}{S+K}} \\
& +\frac{\left(\frac{\mu_{\max } S x}{S+K_{S}}-D x\right)(a+\exp (-b S)) \mu_{\max } S}{\left(D\left(S_{F}-S\right)-(a+\exp (-b S)) \frac{\mu_{\max } S x}{S+K_{S}}\right)^{2}\left(S+K_{S}\right)}, \\
\frac{\partial^{2} \omega}{\partial x^{2}}= & \frac{2\left(\frac{\mu_{\max S} S}{S+K_{S}}-D\right)(a+\exp (-b S)) \mu_{\max } S}{\left(D\left(S_{F}-S\right)-(a+\exp (-b S)) \frac{\mu_{\max } S x}{S+K_{S}}\right)^{2}\left(S+K_{S}\right)} \\
& +\frac{2\left(\frac{\mu_{\max } S x}{S+K_{S}}-D x\right)(a+\exp (-b S))^{2} \mu_{\max }^{2} S^{2}}{\left(D\left(S_{F}-S\right)-(a+\exp (-b S)) \frac{\mu_{\max } S x}{S+K_{S}}\right)^{3}\left(S+K_{S}\right)^{2}} .
\end{aligned}
$$

When $0 \leq x<x_{\delta C}$, in view of the vector field of system (3) that we have $d S / d x>0$ and $d x / d t<0$, that is

$$
D\left(S_{F}-S\right)-(a+\exp (-b S)) \frac{\mu_{\max } S x}{S+K_{S}}>0, \text { and } \frac{\mu_{\max } S}{S+K_{S}}-D<0,
$$

Then we conclude that $\partial \omega / \partial x<0$ and $\partial^{2} \omega / \partial x^{2}<0$ hold true provided $0 \leq x<x_{\delta C}$.

For the scalar differential equation, according to the Cauchy-Lipschitz theorem with parameters we obtain

$$
\frac{\partial x(S, \zeta)}{\partial \zeta}=\exp \left[\int_{(1-\delta) C_{T}}^{S} \frac{\partial}{\partial x}\left(\frac{Q(z, x(z, \zeta))}{P(z, x(z, \zeta))}\right) d z\right]>0
$$

and

$$
\frac{\partial^{2} x(S, \zeta)}{\partial x^{2}}=\frac{\partial x(S, \zeta)}{\partial x} \int_{(1-\delta) C_{T}}^{S} \frac{\partial^{2}}{\partial x^{2}}\left(\frac{Q(z, x(z, \zeta))}{P(z, x(z, \zeta))}\right) \frac{\partial x(S, \zeta)}{\partial x} d z .
$$

From the above analysis, $\partial^{2} x(S, \zeta) / \partial x^{2}<0$ holds. So $\Phi$ is monotonic increasing and concave on $\left[0, x_{\delta C}\right)$ (Fig.1).

(IV) Since $\Phi(x)$ is an increasing function on $\left[0, x_{\delta C}\right]$ and is a decreasing function on $\left[x_{\delta C},+\infty\right)$, it implies that there exists at least one fixed point for the Poincaré map $\Phi$. If $\Phi\left(x_{\delta C}\right) \leq x_{\delta C}$, notice that $\Phi(0)=\tau>0$, then there is a fixed point $x_{f}$ for $\Phi$ with $0<x_{f} \leq x_{\delta C}$ (Fig.1 (a)). While the concavity property of $\Phi$ means $x_{f}$ is unique on $\left[0, x_{\delta C}\right]$, and no fixed point exists on $\left(x_{\delta C},+\infty\right)$ since $\Phi$ is decreasing. If $\Phi\left(x_{\delta C}\right)>x_{\delta C}$, then the concavity of $\Phi$ implies no fixed point exists on $\left[0, x_{\delta C}\right]$. Moreover, $\Phi$ is decreasing on $\left(x_{\delta C},+\infty\right)$. Thus, there exists at least one fixed point for $\Phi$ on $\left(x_{\delta C},+\infty\right)$ (Fig.1(b) and (c)).

$(V)$ Denote the closure of $\mathbb{D}$ by

$$
\overline{\mathbb{D}}=\left\{(S, x) \mid S \geq 0, x \geq 0, x \leq \frac{D\left(S_{F}-S\right)\left(S+K_{S}\right)}{\mu_{\max } S(a+\exp (-b S))}\right\} .
$$

Under case $\left(A_{1}\right)$ of system $(3)$, we show that $\overline{\mathbb{D}}$ is an invariant set. Denoting

$$
L=x-\frac{D\left(S_{F}-S\right)\left(S+K_{S}\right)}{\mu_{\max } S(a+\exp (-b S))} .
$$

If the inequality

$$
\left[(P(S, x), Q(S, x)) \cdot\left(\frac{\partial L}{\partial S}, 1\right)\right]_{L=0} \leq 0
$$


holds, where $\cdot$ denotes the scalar product of two vectors, then all solutions of system (3) are flowing into the boundary of $\overline{\mathbb{D}}$ which means that $\overline{\mathbb{D}}$ is an invariant set. To achieve this purpose, from inequality $(12)$ we have

$$
\begin{aligned}
\left.V(S)\right|_{L=0} & \doteq\left(D\left(S_{F}-S\right)-(a+\exp (-b S)) \frac{\mu_{\max } S x}{S+K_{S}}\right) \cdot \frac{\partial L}{\partial S}+\frac{\mu_{\max } S x}{S+K_{S}}-D x \\
& =x\left(\frac{\mu_{\max } S}{S+K_{S}}-D\right) .
\end{aligned}
$$

In view of the vector fields, $d x / d t<0$ holds for any $x \in\left(0, x_{\delta C}\right)$, that is $\left.V(S)\right|_{L=0}<0$. Therefore, any solution from $\left((1-\delta+\sigma) C_{T}, x_{k}^{+}\right) \in \mathcal{P}$ satisfies $\lim _{x_{k}^{+} \rightarrow+\infty} g\left(x_{k}^{+}\right)=0$. Besides, this solution will meet at the impulsive set after a finite time, after a single pulse,

$$
\lim _{x_{k}^{+} \rightarrow+\infty} \Phi\left(x_{k}^{+}\right)=\lim _{x_{k}^{+} \rightarrow+\infty}(1-\delta+\sigma) g\left(x_{k-1}^{+}\right)+\tau=\tau .
$$

The results reveal that there exists a horizontal asymptote $x=\tau$ as $x_{k}^{+} \rightarrow+\infty$ for the Poincaré map $\Phi$ (Fig.1). This completes the proof.

The main properties of the Poincare map have been studied which play key roles in discussing the existence of the fixed points for $\Phi$, it further enables us to show the existence of order $-k$ periodic solutions of system (3). However, a special case needs to be investigated first.

\subsection{Microorganism eradication periodic solution}

If $x(t)=0$ for system (3), then the microorganism is eradicated and there is a microorganism eradication periodic solution if and only if $\tau=0$. So system (3) is reduced to the following subsystem:

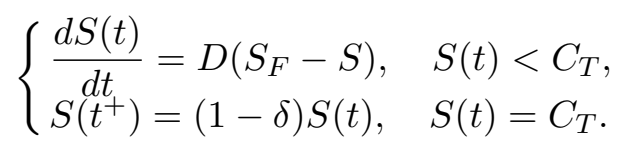

Solving system (13) with initial condition $S\left(0^{+}\right)=(1-\delta) C_{T}$ yields

$$
S(t)=\frac{(1-\delta) C_{T}-S_{F}(1-\exp (D t))}{\exp (D t)},
$$

and the time taken for $S(t)$ to rebound to $C_{T}$ is assumed to be $T$, then

$$
C_{T}=\frac{(1-\delta) C_{T}-S_{F}(1-\exp (D T))}{\exp (D T)} .
$$

By calculation we get the expression of $T$,

$$
T=\frac{1}{D} \ln \left(\frac{(1-\delta) C_{T}-S_{F}}{C_{T}-S_{F}}\right) .
$$

Thus, we obtain a $T$-period microorganism eradication periodic solution $\left(S^{T}(t), x^{T}(t)\right)$ of system $(3)$, where

$$
\left\{\begin{array}{l}
S^{T}(t)=\frac{(1-\delta) C_{T}-S_{F}(1-\exp (D(t-(k-1) T)))}{\exp (D(t-(k-1) T))}, \\
x^{T}(t)=0 .
\end{array}\right.
$$

Under certain conditions, the microorganism eradication periodic solution is stable.

Theorem 2. The microorganism eradication periodic solution $\left(S^{T}(t), 0\right)$ of system (3) is orbitally asymptotically stable provided that

$$
\begin{aligned}
0<\sigma & <\frac{(1-\delta) C_{T}-S_{F}}{C_{T}-S_{F}}\left(\frac{C_{T}+K_{S}}{(1-\delta) C_{T}+K_{S}}\right)^{\frac{\mu_{\max }}{D}}\left(\frac{\left((1-\delta) C_{T}+K_{S}\right)\left(C_{T}-S_{F}\right)}{\left(K_{S}+C_{T}\right)\left((1-\delta) C_{T}-S_{F}\right)}\right)^{\frac{\mu_{\max } S_{F}}{D\left(S_{F}+K_{S}\right)}}-1+\delta \\
& \triangleq \sigma^{*}
\end{aligned}
$$


Proof. To show the stability of the microorganism eradication periodic solution, we need to resort to Lemma 2.1, taking the same notation as for Lemma 2.1,

$$
\begin{aligned}
& P(S, x)=D\left(S_{F}-S\right)-(a+\exp (-b S)) \frac{\mu_{\max } S x}{S+K_{S}}, \quad Q(S, x)=\frac{\mu_{\max } S x}{S+K_{S}}-D x, \\
& \alpha(S, x)=-\delta S, \quad \beta(S, x)=(\sigma-\delta) x+\tau, \quad \phi(S, x)=S-C_{T}, \\
& \left(S^{T}(T), x^{T}(T)\right)=\left(C_{T}, 0\right), \quad\left(S^{T}\left(T^{+}\right), x^{T}\left(T^{+}\right)\right)=\left((1-\delta) C_{T}, 0\right) .
\end{aligned}
$$

Their partial derivatives take the form

$$
\begin{aligned}
& \frac{\partial P}{\partial S}=-D+\frac{b \exp (-b S) \mu_{\max } S x}{S+K_{S}}-\frac{(a+\exp (-b S)) \mu_{\max } x}{S+K_{S}}+\frac{(a+\exp (-b S)) \mu_{\max } S x}{\left(S+K_{S}\right)^{2}} \\
& \frac{\partial Q}{\partial x}=\frac{\mu_{\max } S}{S+K_{S}}-D \\
& \frac{\partial \alpha}{\partial S}=-\delta, \frac{\partial \beta}{\partial x}=\sigma-\delta, \frac{\partial \phi}{\partial S}=1, \frac{\partial \alpha}{\partial x}=\frac{\partial \beta}{\partial S}=\frac{\partial \phi}{\partial x}=0
\end{aligned}
$$

and

$$
\begin{aligned}
\Delta_{1} & =\frac{P_{+}\left(\frac{\partial \beta}{\partial x} \frac{\partial \phi}{\partial S}-\frac{\partial \beta}{\partial S} \frac{\partial \phi}{\partial x}+\frac{\partial \phi}{\partial S}\right)+Q+\left(\frac{\partial \alpha}{\partial S} \frac{\partial \phi}{\partial x}-\frac{\partial \alpha}{\partial x} \frac{\partial \phi}{\partial S}+\frac{\partial \phi}{\partial x}\right)}{P \frac{\partial \phi}{\partial S}+Q \frac{\partial \partial}{x}} \\
& =\frac{P^{+}\left(S^{T}\left(T^{+}\right), x^{T}\left(T^{+}\right)\right)\left(1+\frac{\partial \beta}{\partial x}\right)}{P\left(S^{T}(T), x^{T}(T)\right)}=\frac{(1+\sigma-\delta)\left(S_{F}-(1-\delta) C_{T}\right)}{S_{F}-C_{T}} .
\end{aligned}
$$

Further

$$
\begin{aligned}
& \exp \left(\int_{0}^{T}\left[\frac{\partial P}{\partial S}\left(S^{T}(t), x^{T}(t)\right)+\frac{\partial Q}{\partial x}\left(S^{T}(t), x^{T}(t)\right)\right] d t\right) \\
& =\exp \left(\int_{0}^{T}\left[-2 D+\frac{\mu_{\max } S^{T}(t)}{S^{T}(t)+K_{S}}\right] d t\right) \\
& =\exp \left(-2 D T-\left.\frac{\mu_{\max }}{D} \ln (\exp (-D t)+C)\right|_{0} ^{T}+\left.\frac{\mu_{\max S_{F}}}{D\left(S_{F}+K_{S}\right)} \ln \left(\exp (D t)+\frac{1}{C}\right)\right|_{0} ^{T}\right),
\end{aligned}
$$

where $C=\left(S_{F}+K_{S}\right) /\left((1-\delta) C_{T}-S_{F}\right)$, then

$$
\begin{aligned}
& \exp \left(\int_{0}^{T}\left[\frac{\partial P}{\partial S}\left(S^{T}(t), x^{T}(t)\right)+\frac{\partial Q}{\partial x}\left(S^{T}(t), x^{T}(t)\right)\right] d t\right) \\
& =\left(\frac{C_{T}+K_{S}}{(1-\delta) C_{T}+K_{S}}\right)^{-\frac{\mu_{\max }}{D}}\left(\frac{\left(K_{S}+C_{T}\right)\left((1-\delta) C_{T}-S_{F}\right)}{\left((1-\delta) C_{T}+K_{S}\right)\left(C_{T}-S_{F}\right)}\right)^{\frac{\mu_{\max } S_{F}}{D\left(S_{F}+K_{S}\right)}}\left(\frac{(1-\delta) C_{T}-S_{F}}{C_{T}-S_{F}}\right)^{-2} .
\end{aligned}
$$

By calculation, we get the expression for the Floquet multiplier $\mu_{2}$,

$$
\begin{aligned}
\mu_{2} & =\Delta_{1} \exp \left(\int_{0}^{T}\left[\frac{\partial P}{\partial S}\left(S^{T}(t), x^{T}(t)\right)+\frac{\partial Q}{\partial x}\left(S^{T}(t), x^{T}(t)\right)\right] d t\right) \\
& =\frac{(1+\sigma-\delta)\left(C_{T}-S_{F}\right)}{(1-\delta) C_{T}-S_{F}}\left(\frac{C_{T}+K_{S}}{(1-\delta) C_{T}+K_{S}}\right)^{-\frac{\mu_{\max }}{D}}\left(\frac{\left(K_{S}+C_{T}\right)\left((1-\delta) C_{T}-S_{F}\right)}{\left((1-\delta) C_{T}+K_{S}\right)\left(C_{T}-S_{F}\right)}\right)^{\frac{\mu_{\max } S_{F}}{D\left(S_{F}+K_{S}\right)}} .
\end{aligned}
$$

It follows from (15) that $\left|\mu_{2}\right|<1$, which indicates that the microorganism eradication periodic solution $\left(S^{T}(t), 0\right)$ of system $(3)$ is orbitally asymptotically stable. This completes the proof.

If $\tau=0$, then there is an orbitally asymptotically stable microorganism eradication periodic solution $\left(S^{T}(t), 0\right)$ provided $\sigma<\sigma^{*}$. In this case, the microorganism population was eradicated, which was unexpectated, and it indicates that the application of the control policy failed. In fact, many factors could affect the growth of microorganisms, so we need to identify the key factors that affect the outcomes of the control strategy. It follows from (15) that $\mu_{2}$ depends on each parameter value of model (3). Among these parameters, we need to carry out uncertainty and sensitivity analysis to see which parameter affects the stability of the microorganism eradication periodic solution most, and then put forward a reasonable control strategy related to the key factors. By using the latin hypercube sampling (LHS) method, the PRCCs for various input parameters against the condition $\mu_{2}$ were evaluated with 3,000 samples. A uniform distribution function was employed to address the significance of PRCCs for each parameter of system (3) with wide ranges (Fig.2). The results reveal that the parameters $S_{F}, \mu_{\max }$ and $\sigma$ have positive effects on increasing the value of $\mu_{2}$, while the factors $D, a, b, K_{S}, C_{T}$ and $\delta$ have negative effects on decreasing the value of $\mu_{2}$. Particularly, the value of $\mu_{2}$ is very sensitive to parameters $\delta$ or $\sigma$, and small changes of $\delta$ can lead to microbial extinction, while small changes of $\sigma$ can lead to microbial growth. In contrast, parameters $D, S_{F}, a, b, \mu_{\max }, K_{S}$ and $C_{T}$ have moderate or little effects on the value of $\mu_{2}$. Therefore, the 


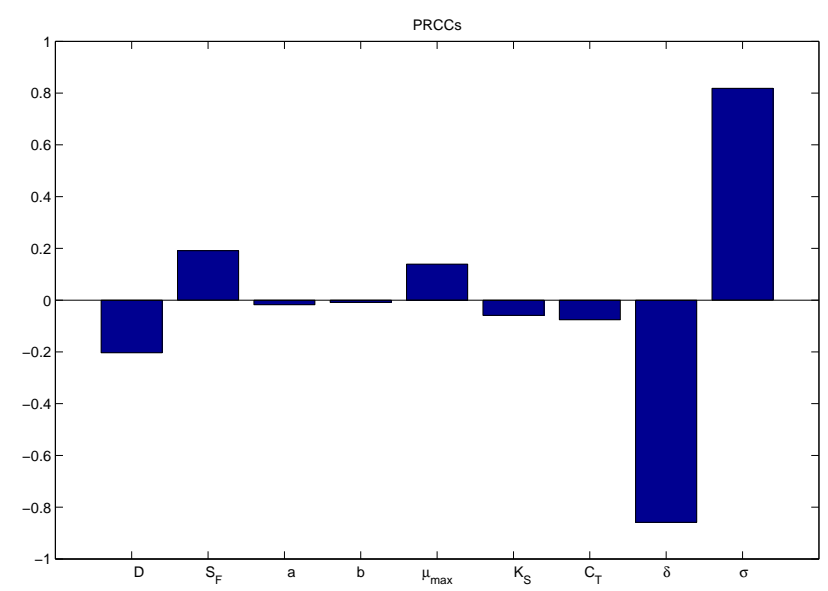

Fig. 2. PRCC results for $\mu_{2}$, the baseline parameters are fixed as: $D=0.15, S_{F}=6, a=0.1, b=0.5, \mu_{\max }=0.3, K_{S}=0.8$, $C_{T}=0.7, \delta=0.14, \sigma=0.15$ and $\tau=0$.

microbial growth or extinction is greatly influenced by these factors, and the parameters that characterize the strength of the control strategy need to be chosen carefully for different purposes.

However, what would happen if $\sigma=\sigma^{*}$, in this case we show that the microorganism eradication periodic solution $\left(S^{T}(t), 0\right)$ becomes unstable and a transcritical bifurcation occurs at $\sigma=\sigma^{*}$.

\subsection{Transcritical bifurcation}

From equation (7), if $\tau=0$, then the Poincaré map $\Phi\left(x_{i}^{+}\right)$can be rewritten as

$$
u \rightarrow(1-\delta+\sigma) f(u) \equiv F(u, \sigma) .
$$

where $u=x_{i}^{+}$and $x \geq 0$ are small enough.

It follows from the Cauchy-Lipschitz theorem that $f(0)=0$, which means that the zero fixed point of Eq.(17) is just the microorganism eradication periodic solution. From Theorem 3.1, it has been shown that the Poincaré map is continuously differentiable with parameters for case $\left(A_{1}\right)$, and so is the function $F(u, \sigma)$ with both $u$ and $\sigma$. Thus, $\lim _{u \rightarrow 0^{+}} f(u)=f(0)=0$. By verifying the conditions of Lemma 2.2 , we have the following conclusions.

Theorem 3. Considering map (17) under case $\left(A_{1}\right)$ (i.e., $\left.C_{T}<S^{*}\right)$, a transcritical bifurcation occurs at $\sigma=\sigma^{*}$, and further there exists a stable order-1 periodic solution of system (3) when $\sigma \in\left(\sigma^{*}, \sigma^{*}+\varepsilon\right)$ with $\varepsilon>0$, where $\sigma^{*}$ is defined by (15).

Proof. For the transcritical bifurcation, it follows from Lemma 2.2 that we only need to verify four corresponding conditions. To do this, the values of $f^{\prime}(u)$ and $f^{\prime \prime}(u)$ at $u=0$ are needed to be calculated first. The solution with initial point $\left((1-\delta) C_{T}, u\right)$ intersects the line $l_{4}$ at a point $\left(C_{T}, f(u)\right)$ (where $0 \leq u \leq u_{0}$, $\left.u_{0} \in D_{0}\right)$. According to the vector fields of system (3), $d x / d t>0$ holds true for any solution that lies below the line $l_{2}$, so system (3) without control can be written by

$$
\frac{d x}{d S}=\frac{Q(S, x)}{P(S, x)} .
$$

where $Q(S, x), P(S, x)$ are defined by Eq. (8). Denote $\left(S, x\left(S ; S_{0}, x_{0}\right)\right)$ be an orbit of system (18), and let $S_{0}=(1-\delta) C_{T}, x_{0}=u, 0 \leq u \leq u_{0}$, thus

$$
x\left(S ;(1-\delta) C_{T}, u\right)=x(S, u), 0 \leq u \leq u_{0},(1-\delta) C_{T} \leq S \leq C_{T} .
$$

Therefore,

$$
\frac{\partial x(S, u)}{\partial u}=\exp \left[\int_{(1-\delta) C_{T}}^{S} \frac{\partial}{\partial x}\left(\frac{Q(s, x(s, u))}{P(s, x(s, u))}\right) d s\right]
$$


and

$$
\frac{\partial^{2} x(S, u)}{\partial u^{2}}=\frac{\partial x(S, u)}{\partial u} \exp \left[\int_{(1-\delta) C_{T}}^{S} \frac{\partial^{2}}{\partial x^{2}}\left(\frac{Q(s, x(s, u))}{P(s, x(s, u))}\right) \frac{\partial x(S, u)}{\partial u} d s\right] .
$$

Notice that $\partial x(S, u) / \partial u>0$, by simple calculation we get $f^{\prime}(0)$, where

$$
\begin{aligned}
f^{\prime}(0) & =\frac{\partial x\left(C_{T}, 0\right)}{\partial u}=\exp \left[\int_{(1-\delta) C_{T}}^{C_{T}} \frac{\partial}{\partial x}\left(\frac{Q(s, x(s, 0))}{P(s, x(s, 0))}\right) d s\right] \\
& =\exp \left[\int_{(1-\delta) C_{T}}^{C_{T}} \frac{\mu_{\max } s}{D\left(s+K_{S}\right)\left(S_{F}-s\right)} d s-\int_{(1-\delta) C_{T}}^{C_{T}} \frac{1}{S_{F}-s} d s\right] \\
& =\frac{\left(C_{T}-S_{F}\right)}{(1-\delta) C_{T}-S_{F}}\left(\frac{C_{T}+K_{S}}{(1-\delta) C_{T}+K_{S}}\right)^{-\frac{\mu_{\max }}{D}}\left(\frac{\left(K_{S}+C_{T}\right)\left((1-\delta) C_{T}-S_{F}\right)}{\left((1-\delta) C_{T}+K_{S}\right)\left(C_{T}-S_{F}\right)}\right)^{\frac{\mu_{\max } S_{F}}{D\left(S_{F}+K_{S}\right)}} .
\end{aligned}
$$

In addition,

$$
f^{\prime \prime}(0)=f^{\prime}(0) \int_{(1-\delta) C_{T}}^{C_{T}} \psi(s) \frac{\partial x(S, 0)}{\partial u} d s
$$

where

$$
\psi(s)=\frac{2 \mu_{\max } s\left(\mu_{\max } s-D\left(s+K_{S}\right)\right)(a+\exp (-b s))}{\left(D\left(S_{F}-s\right)\left(s+K_{S}\right)\right)^{2}}, \quad s \in\left[(1-\delta) C_{T}, C_{T}\right] .
$$

Obviously, $\psi(s)<0$ when $s<S^{*}$, i.e., under case $\left(A_{1}\right)$ we have $f^{\prime \prime}(0)<0$.

On the basis of the above preparations, the conditions of lemma 2.2 can be verified in turn.

(1) The Cauchy-Lipschitz theorem indicates $f(0)=0$, so $F(0, \sigma)=(1-\delta+\sigma) f(0)=0$;

(2) For the value of $\partial F\left(0, \sigma^{*}\right) / \partial u$, we only need to get $\partial F(0, \sigma) / \partial u$, and simple calculation yields

$$
\begin{aligned}
\frac{\partial F(0, \sigma)}{\partial u} & =(1-\delta+\sigma) f^{\prime}(0) \\
& =\frac{(1+\sigma-\delta)\left(C_{T}-S_{F}\right)}{(1-\delta) C_{T}-S_{F}}\left(\frac{C_{T}+K_{S}}{(1-\delta) C_{T}+K_{S}}\right)^{-\frac{\mu_{\max }}{D}}\left(\frac{\left(K_{S}+C_{T}\right)\left((1-\delta) C_{T}-S_{F}\right)}{\left((1-\delta) C_{T}+K_{S}\right)\left(C_{T}-S_{F}\right)}\right)^{\frac{\mu_{\max } S_{F}}{D\left(S_{F}+K_{S}\right)}},
\end{aligned}
$$

according to (15), substitute $\sigma^{*}$ into the above equation, then

$$
\frac{\partial F\left(0, \sigma^{*}\right)}{\partial u}=1,
$$

therefore, $\left(0, \sigma^{*}\right)$ is a fixed point of $F(u, \sigma)$, and its eigenvalue is 1 ;

(3) The third condition satifies

$$
\frac{\partial^{2} F\left(0, \sigma^{*}\right)}{\partial u \partial \sigma}=\frac{\partial\left(f^{\prime}(0)(1-\delta+\sigma)\right)}{\partial \sigma}=f^{\prime}(0)>0,
$$

(4) At last, we have shown $f^{\prime \prime}(0)<0$ for case $\left(A_{1}\right)$, thus,

$$
\frac{\partial^{2} F\left(0, \sigma^{*}\right)}{\partial u^{2}}=\left(1-\delta+\sigma^{*}\right) f^{\prime \prime}(0)<0 .
$$

In conclusion, four conditions of Lemma 2.2 for the transcritical bifurcation have been verified, which implies that an order-1 periodic solution is generated for system (3) provided $\sigma \in\left(\sigma^{*}, \sigma^{*}+\varepsilon\right)$. This completes the proof.

The results of Theorem 3.2 and Theorem 3.3 suggest that the microorganism eradication periodic solution is orbitally asymptotically stable provided $\sigma<\sigma^{*}$, a transcritical bifurcation occurs at $\sigma=\sigma^{*}$ and then an order- 1 periodic solution is generated, as shown in Fig.3, where $\sigma^{*} \approx 0.1521$. Furthermore, in order to address how the control parameter $\sigma$ affects the complex dynamics of system (3) as $\sigma$ increases, bifurcation diagrams of $\sigma$ were plotted (Fig.4). When $\sigma>\sigma^{*}$, the order-1 periodic solution losses stability when $\sigma$ passes through a critical value, and an order-2 periodic solution is generated. As $\sigma$ increases, period-doubling bifurcations lead system (3) to chaos. When $\sigma$ increases further, model (3) exhibits sharp transitions from order- $(n+1)$ periodic solutions to order- $n(n=1,2, \cdots, 5)$ periodic solutions via perioddecreasing bifurcations. It indicates not only that the microbial growth is very sensitive to $\sigma$, but also that system (3) exhibits very complex dynamics when $\tau=0$. The aim of the coming section is to provide comprehensive qualitative analysis for the complex dynamics of system (3) when $\tau>0$. 
(a)
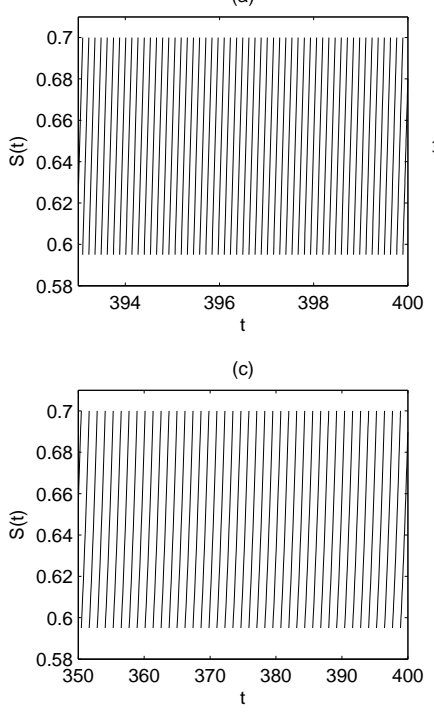

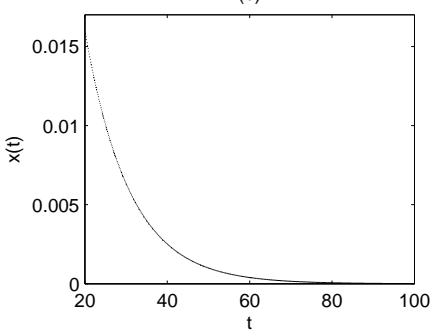

(d)

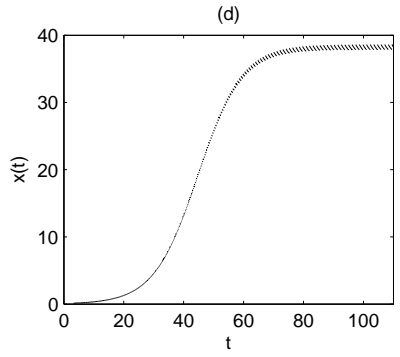

Fig. 3. (a-c) Stable microorganism eradication periodic solution when $\sigma=0.14$; (e-f) Stable order-1 periodic solution with $\sigma=0.17$. All other parameters are fixed as $D=0.15, S_{F}=6, a=0.1, b=0.5, \mu_{\max }=0.3, K_{S}=0.8, C_{T}=0.7, \delta=0.15$ and $\tau=0$.
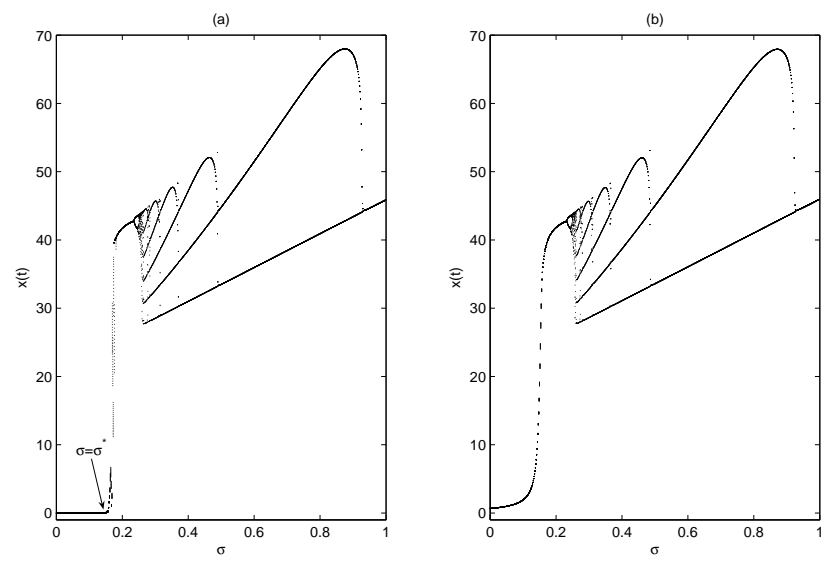

Fig. 4. (a) Bifurcation diagram for case $\left(A_{1}\right)$ with respect to $\sigma$ when $\tau=0$; (b) Bifurcation diagram for case $\left(A_{1}\right)$ with with respect to $\sigma$ when $\tau=0.1>0$. All other parameters are fixed as: $D=0.15, S_{F}=6, a=0.1, b=0.5, \mu_{\max }=0.3, K_{S}=0.8$, $C_{T}=0.7$ and $\delta=0.15$.

\subsection{Order-k periodic solutions for $\tau>0$}

There are two aspects to be addressed in this section. The first question involves the existence of the order- $k(k \geq 1)$ periodic solutions. The second problem relates to numerical investigations of the complex dynamics of system (3).

For case $\left(A_{1}\right)$, any solution initiating from the phase set experiences impulsive effects, after $n(n=$ $1,2, \cdots)$ times pulses, it follows from the definition of the Poincaré map that we denote the corresponding impulsive point series as $x_{n}^{+}=\Phi^{n}\left(x_{0}^{+}\right)$. In view of Theorem 3.1, there always exists a fixed point for the Poincaré map. Denote the order-1 periodic solution as $(\xi(t), \eta(t))$, and then we show the generalised conditions for stability of it by employing Lemma 2.1.

Theorem 4. The order-1 periodic solution $(\xi(t), \eta(t))$ of system (3) is orbitally asymptotically stable if and 
only if

$$
\left|\frac{P^{+}\left((1-\delta) C_{T},(1+\sigma-\delta) \eta_{0}+\tau\right)(1+\sigma-\delta)}{P\left(C_{T}, \eta_{0}\right)} \exp \left(\int_{0}^{T}\left[\frac{\partial P}{\partial S}(\xi(t), \eta(t))+\frac{\partial Q}{\partial x}(\xi(t), \eta(t))\right] d t\right)\right|<1 .
$$

Proof. Assume that the initial point and end point of the order-1 periodic solution $(\xi(t), \eta(t))$ are denoted by $M\left(C_{T}, \eta_{0}\right)$ and $M^{+}\left((1-\delta) C_{T},(1+\sigma-\delta) \eta_{0}+\tau\right)$, respectively. Taking the same notation and techniques as used in the proof of Theorem 3.2, the Floquet multiplier $\mu_{2}$ can be calculated,

$$
\mu_{2}=\frac{P^{+}\left((1-\delta) C_{T},(1+\sigma-\delta) \eta_{0}+\tau\right)(1+\sigma-\delta)}{P\left(C_{T}, \eta_{0}\right)} \exp \left(\int_{0}^{T}\left[\frac{\partial P}{\partial S}(\xi(t), \eta(t))+\frac{\partial Q}{\partial x}(\xi(t), \eta(t))\right] d t\right) .
$$

where $P, Q, \frac{\partial P}{\partial S}, \frac{\partial Q}{\partial x}$ have the same expressions as those shown in Theorem 3.2. The condition of Theorem 3.4 guarantees $\left|\mu_{2}\right|<1$, so the order-1 periodic solution $(\xi(t), \eta(t))$ is orbitally asymptotically stable. This completes the proof.

Theorem 5. If $\Phi\left(x_{\delta C}\right)<x_{\delta C}$, then for the Poincaré map $\Phi$ there exists a globally asymptotically stable fixed point $x_{f}$ with $0<x_{f} \leq x_{\delta C}$, which corresponds to a stable order-1 periodic solution of system (3).

Proof. According to the property (IV) of Theorem 3.1 of the Poincaré map, it has been proved that the Poincaré map has a unique fixed point $x_{f}$ with $0<x_{f} \leq x_{\delta C}$ provided $\Phi\left(x_{\delta C}\right)<x_{\delta C}$. Theorem 3.4 has provided the conditions for the orbitally asymptotically stability of this fixed point. Thus, to show the global stability of it, we only need to show it is globally attractive.

For any point $\left((1-\delta) C_{T}, x_{0}^{+}\right) \in \mathcal{P}$, if $x_{0}^{+} \in\left[0, x_{f}\right)$, then the properties (II) and (III) of the Poincaré map lead to $x_{f}>\Phi\left(x_{0}^{+}\right)>x_{0}^{+}$. After $n$ pulses, $\Phi^{n}\left(x_{0}^{+}\right)$is monotonically increasing as $n$ increases and $\lim _{n \rightarrow+\infty} \Phi^{n}\left(x_{0}^{+}\right)=x_{f}$ (Fig. 1 (a)).

If $x_{0}^{+}>x_{f}$, then two possible cases of $\Phi^{n}\left(x_{0}^{+}\right)$need to be considered: (a) $\Phi^{n}\left(x_{0}^{+}\right)>x_{f}$ for all $n$; (b) $\Phi^{n}\left(x_{0}^{+}\right)>x_{f}$ does not hold true for all $n$. For the former, the condition $\Phi\left(x_{0}^{+}\right)<x_{0}^{+}$implies that $\Phi^{n}\left(x_{0}^{+}\right)$is monotonically decreasing and $\lim _{n \rightarrow+\infty} \Phi^{n}\left(x_{0}^{+}\right)=x_{f}$. For the latter, without loss of generality we assume that there is a smallest positive integer $n_{1}$ such that $\Phi^{n_{1}}\left(x_{0}^{+}\right)<x_{f}$. From case $(a), \Phi^{n_{2}}\left(y_{0}^{+}\right)$is monotonically increasing as $n_{2}$ increases $\left(n_{2}\right.$ is a positive integer and $\left.n_{2}>n_{1}\right)$ and $\lim _{n_{2} \rightarrow+\infty} \Phi^{n_{2}}\left(x_{0}^{+}\right)=x_{f}$. It follows from the above discussions that the unique order- 1 periodic solution is globally attracting, which indicates it is globally asymptotically stable. This completes the proof.

Remark 1. Particularly, there is a special case for Theorem 3.5, if $\Phi\left(x_{\delta C}\right)=x_{\delta C}$, then $x=x_{\delta C}$ is a globally asymptotically stable fixed point for the Poincaré map, which means that for system (3) there exists a globally asymptotically stable order-1 periodic solution.

Theorem 6. If $\Phi\left(x_{\delta C}\right)>x_{\delta C}$ and $\Phi^{2}\left(x_{\delta C}\right) \geq x_{\delta C}$, then for the Poincaré map $\Phi$ there exists a stable fixed point or a stable fixed point with period two, which indicates that there only exists a stable order-1 periodic solution or order-2 periodic solution of system (3).

Proof. According to the property (I) of Theorem 3.1, if $\Phi\left(x_{\delta C}\right)>x_{\delta C}$, then on $\left[0, x_{\delta C}\right]$ the Poincaré map $\Phi$ not only is monotonically increasing, but also does not exist as a fixed point. So there must be a positive integer $i$ such that $x_{i-1}^{+}<x_{\delta C}, x_{i}^{+} \geq x_{\delta C}$ and $x_{i}^{+}=\Phi\left(x_{i-1}^{+}\right) \leq \Phi\left(x_{\delta C}\right)$, that is to say $x_{i}^{+} \in\left[x_{\delta C}, \Phi\left(x_{\delta C}\right)\right]$. Since the Poincaré map $\Phi$ is monotonically decreasing on $\left[x_{\delta C},+\infty\right)$, after a single impulsive effect we have $x_{1}^{+}=\Phi\left(x_{0}^{+}\right) \leq \Phi\left(x_{\delta C}\right)$. Therefore, there is a positive integer $i \geq 1$ such that $x_{i}^{+} \in\left[x_{\delta C}, \Phi\left(x_{\delta C}\right)\right]$.

It is found that the set $\left[x_{\delta C}, \Phi\left(x_{\delta C}\right)\right]$ is an invariant set. In fact, according to Theorem $3.1, \Phi$ is monotonically decreasing on $\left[x_{\delta C},+\infty\right)$ and $\Phi^{2}$ is monotonically increasing on $\left[x_{\delta C},+\infty\right)$, so

$$
\Phi\left(\left[x_{\delta C}, \Phi\left(x_{\delta C}\right)\right]\right)=\left[\Phi^{2}\left(x_{\delta C}\right), \Phi\left(x_{\delta C}\right)\right] \subset\left[x_{\delta C}, \Phi\left(x_{\delta C}\right)\right] .
$$

Without loss of generality, we assume $x_{1}^{+}=\Phi\left(x_{0}^{+}\right) \neq x_{0}^{+}, x_{2}^{+}=\Phi^{2}\left(x_{0}^{+}\right) \neq x_{0}^{+}$and $x_{n}^{+}=\Phi^{n}\left(x_{0}^{+}\right)$for any $x_{0}^{+} \in\left[x_{\delta C}, \Phi\left(x_{\delta C}\right)\right]$. For the relations among $x_{\delta C}, \Phi\left(x_{\delta C}\right), x_{0}^{+}, x_{1}^{+}$and $x_{2}^{+}$, there only exist four possible cases: 
$\left(C_{1}\right) x_{\delta C} \leq x_{2}^{+}<x_{0}^{+}<x_{1}^{+} \leq \Phi\left(x_{\delta C}\right)$. It is observed that $x_{1}^{+}=\Phi\left(x_{0}^{+}\right)<\Phi\left(x_{2}^{+}\right)=x_{3}^{+}$and $x_{4}^{+}=$ $\Phi\left(x_{3}^{+}\right)<\Phi\left(x_{1}^{+}\right)=x_{2}^{+}$, so $x_{4}^{+}<x_{2}^{+}<x_{0}^{+}<x_{1}^{+}<x_{3}^{+}$. By induction we have

$$
\begin{aligned}
& x_{\delta C} \leq \cdots<x_{2 n+2}^{+}<x_{2 n}^{+}<\cdots<x_{2}^{+}<x_{0}^{+} \\
& <x_{1}^{+}<\cdots<x_{2 n-1}^{+}<x_{2 n+1}^{+}<\cdots \leq \Phi\left(x_{\delta C}\right) .
\end{aligned}
$$

$\left(C_{2}\right) x_{\delta C} \leq x_{0}^{+}<x_{2}^{+}<x_{1}^{+} \leq \Phi\left(x_{\delta C}\right)$. Similarly we have $\Phi\left(x_{1}^{+}\right)=x_{2}^{+}<x_{3}^{+}=\Phi\left(x_{2}^{+}\right)<\Phi\left(x_{0}^{+}\right)=x_{1}^{+}$ and $x_{2}^{+}=\Phi\left(x_{1}^{+}\right)<\Phi\left(x_{3}^{+}\right)=x_{4}^{+}<x_{3}^{+}=\Phi\left(x_{2}^{+}\right)=x_{1}^{+}$, thus $x_{0}^{+}<x_{2}^{+}<x_{4}^{+}<x_{3}^{+}<x_{1}^{+}$. By induction one obtains

$$
\begin{aligned}
& x_{\delta C} \leq x_{0}^{+}<x_{2}^{+}<\cdots<x_{2 n}^{+}<x_{2 n+2}^{+} \\
& <\cdots<x_{2 n+1}^{+}<x_{2 n-1}^{+}<\cdots<x_{1}^{+} \leq \Phi\left(x_{\delta C}\right) .
\end{aligned}
$$

$\left(C_{3}\right) x_{\delta C} \leq x_{1}^{+}<x_{2}^{+}<x_{0}^{+} \leq \Phi\left(x_{\delta C}\right)$. By using the same analytical techniques as case $\left(C_{2}\right)$ one obtains

$$
\begin{aligned}
& x_{\delta C} \leq x_{1}^{+}<\cdots<x_{2 n-1}^{+}<x_{2 n+1}^{+}<\cdots \\
& <x_{2 n+2}^{+}<x_{2 n}^{+}<\cdots<x_{2}^{+}<x_{0}^{+} \leq \Phi\left(x_{\delta C}\right) .
\end{aligned}
$$

$\left(C_{4}\right) x_{\delta C} \leq x_{1}^{+}<x_{0}^{+}<x_{2}^{+} \leq \Phi\left(x_{\delta C}\right)$. By using the same analytical techniques as case $\left(C_{1}\right)$ one obtains

$$
\begin{aligned}
& x_{\delta C} \leq \cdots<x_{2 n+1}^{+}<x_{2 n-1}^{+}<\cdots<x_{1}^{+}<x_{0}^{+} \\
& <x_{2}^{+}<\cdots<x_{2 n}^{+}<x_{2 n+2}^{+}<\cdots \leq \Phi\left(x_{\delta C}\right) .
\end{aligned}
$$

It follows from cases $\left(C_{2}\right)$ and $\left(C_{3}\right)$ that there may be a unique $x_{f}$ such that

$$
\lim _{n \rightarrow+\infty} x_{2 n+1}^{+}=\lim _{n \rightarrow+\infty} x_{2 n}^{+}=x_{f},\left(x_{f} \in\left[x_{\delta C}, \Phi\left(x_{\delta C}\right)\right]\right),
$$

or there may be two fixed points $x_{f}^{1}$ and $x_{f}^{2}$ such that

$$
\lim _{n \rightarrow+\infty} x_{2 n+1}^{+}=x_{f}^{1} \quad \text { and } \lim _{n \rightarrow+\infty} x_{2 n}^{+}=x_{f}^{2},
$$

where $x_{f}^{1}, x_{f}^{2} \in\left[x_{\delta C}, \Phi\left(x_{\delta C}\right)\right]$ and $x_{f}^{1} \neq x_{f}^{2}$. There results reveal that there either exists a unique order-1 periodic solution or order-2 periodic solution in system (3). Furthermore, for cases $\left(C_{1}\right)$ and $\left(C_{4}\right)$, there only exists a unique order-2 periodic solution. In conclusion, it suggests that for system (3) order- $k(k \geq 3)$ periodic solutions do not exist other than the order-1 periodic solution or order-2 periodic solution (Fig.1(b) and $(\mathrm{c}))$. This completes the proof.

Theorem 7. Let $x_{m}^{+}=\min \left\{x^{+}: \Phi\left(x^{+}\right)=x_{\delta C}\right\}$. If $\Phi\left(x_{\delta C}\right)>x_{\delta C}$ and $\Phi^{2}\left(x_{\delta C}\right)<x_{m}^{+}$, then there exists an order-3 periodic solution of system (3).

Proof. The results of Theorem 3.1 and Theorem 3.6 suggest that there only exists a unique fixed point $x_{f}$ for the Poincaré map provided $\Phi\left(x_{\delta C}\right)>x_{\delta C}$ on the interval $\left(x_{\delta C}, \Phi\left(x_{\delta C}\right)\right)$. To show the existence of an order-3 periodic solution, we only need to show that there is a $x_{f}^{*} \in[0,+\infty)$ such that $\Phi^{3}\left(x_{f}^{*}\right)=x_{f}^{*}$ and $\Phi\left(x_{f}^{*}\right) \neq x_{f}^{*}$ holds. It is observed that $\Phi^{3}(x)$ is continuous on $[0,+\infty)$ because $\Phi(x)$ is continuous on $[0,+\infty)$. Thus, the Poincaré map $\Phi^{3}$ satisfies

$$
\Phi^{3}\left(x_{m}^{+}\right)=\Phi^{2}\left(x_{\delta C}\right)<x_{m}^{+}, \quad \text { and } \quad \Phi^{3}(0)=\Phi^{2}(\tau)>0,
$$

it indicates that there is a fixed point $x_{f}^{*}$ such that $\Phi^{3}\left(x_{f}^{*}\right)=x_{f}^{*}$ with $x_{f}^{*} \in\left(0, x_{m}^{+}\right), x_{m}^{+}<x_{\delta C}$. In addition, the fixed point $x_{f}>x_{\delta C}$, so $\Phi\left(x_{f}^{*}\right) \neq x_{f}^{*}$. Therefore, for the Poincaré map $\Phi^{3}$ there exists a fixed point which is just an order-3 periodic solution of system (3) (Fig.1(d)). This completes the proof.

Remark 2. Theorem 3.7 has provided simple conditions for the existence of an order-3 periodic solution. Further, Li and York pointed out that an order-3 periodic solution means chaos [Devaney , 2003; Li \& Yorke, 1975].

For case $\left(A_{1}\right)$ with $\tau>0$, the conditions for the global stablility of the order -1 periodic solution have

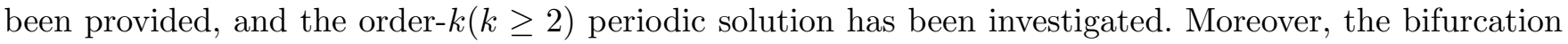
diagrams with respect to control parameters have been plotted, the numerical results support the theoretical 
conclusions, with the parameters fixed as shown in Fig.4(b). When $\sigma>0$, there exists a globally stable order-1 periodic solution. As $\sigma$ increases, an order-2 periodic solution is generated through period-doubling bifurcation, which finally leads system (3) to chaos. As $\sigma$ further increases, system (3) exhibits sharp transitions from order- $(n+1)$ periodic solutions to order- $n(n=1,2, \cdots, 5)$ periodic solutions via perioddecreasing bifurcations. The results reveal not only that for system (3) complex dynamics exist when $\tau>0$, but also that the microbial growth is greatly influenced by the control parameter $\sigma$. Besides, it confirms that small changes in $\sigma$ will cause the substrate and microorganism populations oscillate periodically with distinct amplitudes and periods.

\section{Complexity of the Poincaré map for case $\left(A_{2}\right)$}

\subsection{Complex domains of impulsive and phase sets}

It follows from Lemma 2.3 that the equilibrium $E^{*}$ of system (3) could be stable if $a \geq \theta$ or unstable if $a<\theta$. For case $\left(A_{2}\right)$, as discussed before, if the location of $L_{3}, L_{4}$ or $x^{*}$ changes, then the solution initiating from the phase set may experience infinitely many times pulses, or a finite number of pulses, or will not experience a pulse at all. With so many interesting problems arising, what are the exact domains of the Poincaré map? What are the exact conditions for infinitely many pulses, a finite number of pulses or no pulses? Also, what types of dynamical behaviour of system (3) will be presented? To address these questions, it is necessary to define the exact domains of the impulsive set and phase set. Without loss of generality, unless otherwise specified we assume that $E^{*}$ is always a focus of system (3) for simplicity.

For case $\left(A_{2}\right)$, if $a \geq \theta$, then $E^{*}$ is a stable focus. From the analysis of Section 3 , if $(1-\delta) C_{T}<S_{P}$, then the solution starting from point $I_{P}^{1}\left((1-\delta) C_{T}, x_{\delta C}\right)$ meets $l_{4}$ at point $Q\left(C_{T}, x_{Q}\right)$. Thus, the exact domain of the impulsive set is defined as $\mathcal{I}_{1}=\left\{(S, x) \in R^{2} \mid S=C_{T}, 0 \leq x \leq x_{Q}\right\}$, and the exact domain of the phase set is defined as $\mathcal{P}$. If $(1-\delta) C_{T} \geq S_{P}$, then the exact domain of the impulsive set is defined as $\mathcal{I}$ and the exact domain of the phase set is defined as $\mathcal{P}_{1}=\left\{\left(S^{+}, x^{+}\right) \in R^{2} \mid S^{+}=(1-\delta) C_{T}, x^{+} \in D_{1}\right\}$, where $D_{1}=\left\{\left[x_{\max },+\infty\right) \bigcup\left[0, x_{\min }\right]\right\}$ (Fig.5 (a)).
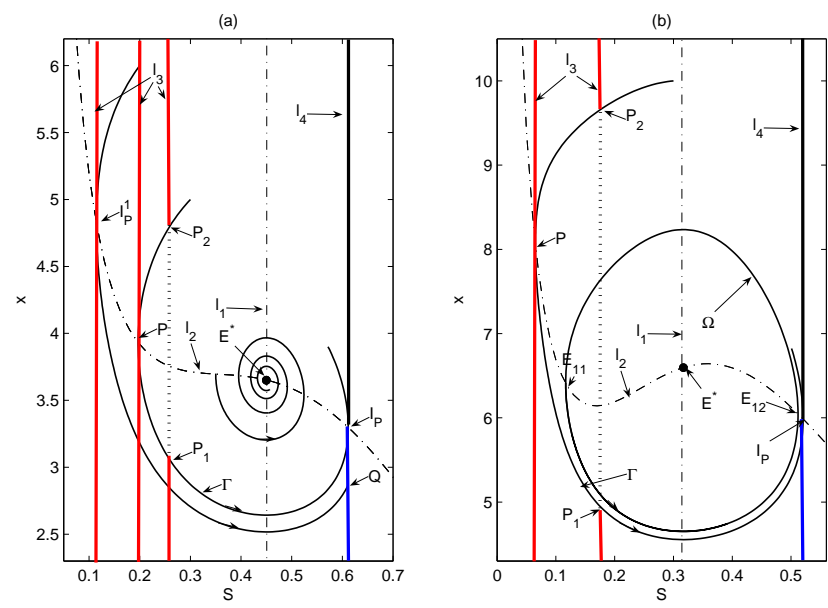

Fig. 5. The exact domains of the phase set and the impulsive set for case $\left(A_{2}\right)$. (a) Case $a \geq \theta$; (b) Case $a<\theta$. The blue lines denote the phase sets and the red lines represent the impulsive sets.

If $a<\theta$, then $E^{*}$ is an unstable focus and there is a unique stable limit cycle $\Omega$. The isocline $l_{2}$ and the limit cycle $\Omega$ have two intersection points, denoted by $E_{11}\left(S_{11}, x_{11}\right)$ and $E_{12}\left(S_{12}, x_{12}\right)$ with $S_{11}<S_{12}$ (Fig.5 (b)). In order to provide the exact domains of the impulsive set and phase set, we need to discuss the following three cases according to the different locations among $(1-\delta) C_{T}, C_{T}, x_{11}$ and $x_{12}$ :

$$
\begin{aligned}
& \left(B_{1}\right)(1-\delta) C_{T} \leq S_{11} \quad \text { and } C_{T}<S_{12}, \\
& \left(B_{2}\right) C_{T} \geq S_{12} \text { and }\left(B_{3}\right) S_{11}<(1-\delta) C_{T}<C_{T}<S_{12} .
\end{aligned}
$$


For case $\left(B_{1}\right)$ (Fig.5 (b)), it follows from case $\left(A_{2}\right)$ for $a \geq \theta$ that the exact domain of the impulsive set can be defined as $\mathcal{I}_{1}$, and the exact domain of the phase set can be defined as $\mathcal{P}$. Similarly, for case $\left(B_{2}\right)$, if $(1-\delta) C_{T}<S_{P}$, the exact domain of the impulsive set can be defined as $\mathcal{I}_{1}$, and the exact domain of the phase set can be defined as $\mathcal{P}$. If $(1-\delta) C_{T} \geq S_{P}$, then the exact domain of the impulsive set can be defined as $\mathcal{I}$ and the exact domain of the phase set can be defined as $\mathcal{P}_{1}$. For case $\left(B_{3}\right)$, parts of $l_{3}$ and $l_{4}$ are lying in the interior of the limit cycle $\Omega$, in view of the vector fields of the system (3), any solution from the phase set $\mathcal{P}$ (except for $E^{*}$ ) may have finite or infinite intersection points with $l_{3}$ before reaching the impulsive set. So the impulsive set can be defined as $\mathcal{I}$ and the phase set is defined as $\mathcal{P} /\left\{E^{*}\right\}$, where $\mathcal{P} /\left\{E^{*}\right\}$ means $E^{*}$ is removed from $\mathcal{P}$.

Table 1. Exact domains of impulsive and phase sets of system (3)) for case $\left(A_{2}\right)$, where Is and Ps denote the impulsive set and phase set, respectively.

\begin{tabular}{cccccc}
\hline Cases & Cases & $C_{T}$ & $(1-\delta) C_{T}$ & Is & Ps \\
\hline$a \geq \theta$ & $S C_{1}$ & $A_{2}$ & $(1-\delta) C_{T}<S_{P}$ & $\mathcal{I}_{1}$ & $\mathcal{P}$ \\
$a \geq \theta$ & $S C_{2}$ & $\left(A_{2}\right)$ & $(1-\delta) C_{T} \geq S_{P}$ & $\mathcal{I}$ & $\mathcal{P}_{1}$ \\
$a<\theta$ & $S C_{1}$ & $\left(B_{1}\right)$ & $(1-\delta) C_{T} \leq S_{11}$ & $\mathcal{I}_{1}$ & $\mathcal{P}$ \\
$a<\theta$ & $S C_{1}$ & $\left(B_{2}\right)$ & $(1-\delta) C_{T}<S_{P}$ & $\mathcal{I}_{1}$ & $\mathcal{P}$ \\
$a<\theta$ & $S C_{2}$ & $\left(B_{2}\right)$ & $(1-\delta) C_{T} \geq S_{P}$ & $\mathcal{I}$ & $\mathcal{P}_{1}$ \\
$a<\theta$ & $S C_{3}$ & $\left(B_{3}\right)$ & $S_{11}<(1-\delta) C_{T}<S_{12}$ & $\mathcal{I}$ & $\mathcal{P} /\left\{E^{*}\right\}$ \\
\hline
\end{tabular}

From the above analysis, for the case $\left(A_{2}\right)$ we have defined all exact domains of impulsive and phase sets of model (3), and the results are listed in Table 4.1. For simplicity, the identical notations are adopted to denote cases which have the same impulsive set and phase set, i.e., $\left(S C_{1}\right)$ is adopted to denote the impulsive set $\mathcal{I}_{1}$ and the phase set $\mathcal{P} ;\left(S C_{2}\right)$ is adopted to denote the impulsive set $\mathcal{I}$ and the phase set $\mathcal{P}_{1}$; $\left(S C_{3}\right)$ is adopted to denote the impulsive set $\mathcal{I}$ and the phase set $\mathcal{P} /\left\{E^{*}\right\}$. Since the domains of the Poincaré map are determined by cases $\left(S C_{i}\right)(i=1,2,3)$, the Poincaré map is well defined and their properties are all satisfied as shown in Theorem 1. Like case $\left(A_{1}\right)$, we can resort to the same analytical methods to study the existence and stability of order- $k$ periodic solutions. To avoid repetition, the analytical results which are different from case $\left(A_{1}\right)$ are discussed.

\subsection{Different results of case $\left(A_{2}\right)$}

Theorem 8. For case $\left(S C_{2}\right)$, if $\Phi\left(x_{\min }\right) \leq x_{\min }$ or $\Phi\left(x_{\min }\right) \geq x_{\max }$, then system (3) has a unique stable order-1 periodic solution. If $\Phi\left(x_{\max }\right)>x_{\max }$ and $\Phi^{2}\left(x_{\max }\right) \geq x_{\max }$, then system (3) has either a stable order-1 periodic solution or an order-2 periodic solution.

Proof. If $\Phi\left(x_{\min }\right)=x_{\min }$ or $\Phi\left(x_{\min }\right)=x_{\max }$, then $x_{\min }$ or $x_{\max }$ is the fixed point of the Poincaré map, which is just the order-1 periodic solution of system (3). If $\Phi\left(x_{\min }\right)<x_{\min }$, then any solution from $\mathcal{P}_{1}$ experiences infinitely many pulses, and the corresponding impulsive point series $x_{n}^{+} \in\left[0, x_{\min }\right)$. Theorem 3.1 reveals that $\Phi$ is monotonically decreasing on $\left[x_{\max },+\infty\right)$ and increasing on $\left[0, x_{\min }\right]$. So there is a unique $x_{f} \in\left[0, x_{\min }\right)$ such that $\lim _{n \rightarrow+\infty} x_{n}^{+}=x_{f}$.

When $\Phi\left(x_{\min }\right)>x_{\max }$, two possible cases need to be discussed: $\tau \geq x_{\max }$ and $\tau<x_{\max }$. For the former, any solution from $\mathcal{P}_{1}$ will experience infinitely many pulses. According to Theorem 3.1, there must be a unique fixed point $x_{f} \in\left(x_{\max },+\infty\right)$. For the latter, there exists a positive $x_{C}$ such that $\Phi\left(x_{C}\right)=x_{\max }$. If $x_{0}^{+} \in\left[0, x_{C}\right) \subset\left[0, x_{\text {min }}\right]$ ( or $x_{0}^{+} \in\left(x_{C}^{1}, x_{C}\right)$ and $0<x_{C}^{1}<x_{C}$ ), after a single pulse, the solution never experiences any more pulses. Thus, on $D_{1} /\left[0, x_{C}\right)$ (or $D_{1} /\left(x_{C}^{1}, x_{C}\right)$ ) the Poincaré map is well defined and the properties as shown in Theorem 1 are all satisfied, which indicates that for system (3) there exists a stable order-1 periodic solution.

If $\Phi\left(x_{\max }\right)>x_{\max }$ and $\Phi^{2}\left(x_{\max }\right) \geq x_{\max }$, then Theorem 3.6 suggests that there either exists a stable order-1 periodic solution or an order- 2 periodic solution. This completes the proof. 
Remark 3. Under case $\left(S C_{2}\right)$, if $x_{\min }<\Phi\left(x_{\min }\right)<x_{\max }$ or $x_{\min }<\Phi\left(x_{\max }\right)<x_{\max }$, then the solution from $\mathcal{P}_{1}$ only experiences a finite number of pulses, and then converges to the invariant set $\left(x_{\min }, x_{\max }\right)$. In particular, if $a \geq \theta,(1-\delta) C_{T} \geq S_{P}, C_{T}>S^{*}$ and $x_{\min }<\Phi\left(x_{\min }\right)<x_{\max }$, then all solutions will finally tend to the stable focus $E^{*}$ after finite pulses. If $a<\theta,(1-\delta) C_{T} \geq S_{P}, C_{T}>S^{*}$ and $x_{\min }<\Phi\left(x_{\min }\right)<x_{\max }$, then all solutions will finally tend to the stable limit cycle $\Omega$ after a finite number of pulses,

The results of Remark 3 show that for system (3) there exists multi-stable behaviors. It suggests that the concentrations of the substrate and microorganism population may stabilize at different fixed levels under different initial concentrations. Furthermore, the final concentrations of the microorganism population mainly depend on its initial concentrations as well as on the ratios of the initial concentrations of the substrate and microorganism populations.
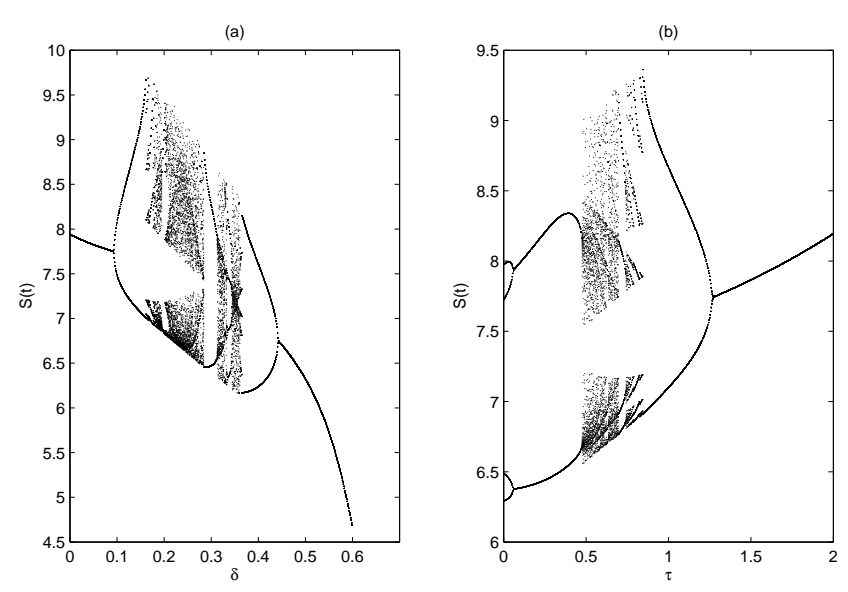

Fig. 6. (a) Bifurcation diagram of case $\left(S C_{3}\right)$ with respect to $\delta$ when $\tau=0.3$ and $\sigma=0.6$; (b) Bifurcation diagram for case $\left(S C_{3}\right)$ with with respect to $\tau$ when $\delta=0.3$ and $\sigma=0.6$. All other parameters are fixed as: $D=0.1, S_{F}=1.5, a=0.1, b=8$, $\mu_{\max }=0.4, K_{S}=0.95$ and $C_{T}=0.4$.

For case $\left(S C_{3}\right)$, parts of the impulsive and phase sets are lying in the interior of the stable limit cycle $\Omega$, and all solutions from the phase set $\mathcal{P} /\left\{E^{*}\right\}$ have pulsed actions. On the one hand, the bifurcation diagrams with respect to $\delta$ and $\tau$ have shown that system (3) presents very complex dynamics, such as period-doubling bifurcations, period-halving bifurcations, crisis and chaotic bands (Fig.6). On the other hand, it is observed that for the Poincaré map there exists complicated shapes with discontinuity points, as shown in Fig.7, where the discontinuity points are denoted by $D C_{i}(i=1,2,3, \cdots)$. As the control parameter $\delta$ increases, the number of discontinuity points first increases and reaches its maximum value around $E^{*}$, and then decreases (Fig.7). Moreover, there is an interesting fact that for the order- $k(k \geq 2)$ periodic solutions there exist discontinuity points (Fig.7 (e), (f) and (g)).

\section{Conclusions}

Recently, mathematical models of the growth of microorganisms in a chemostat with multiple control strategies have become a popular research topic, with studies mainly focussed on the chemostat models with impulsive control [Sun \& Chen , 2007; Sun et al., 2010, 2011; Tian et al., 2010; Li et al., 2009; Yang et al., 2017]. However, these studies only viewed the microorganism concentration as the basis for implementation of control strategies. In fact, the concentration of the substrate which plays an important role in microorganism cultivation can also be considered as another criterion for applying a control strategy [Simkins \& Alexander , 1984, 1985]. Therefore, we proposed a novel impulsive chemostat model with the substrate concentration as the controllable threshold. There are three aspects which need to be addressed, the first question involves the comprehensive analysis of the dynamics of system (3), the second problem relates to providing numerical studies to substantiate our results and the third aspect deals with comparisons with previous studies. 

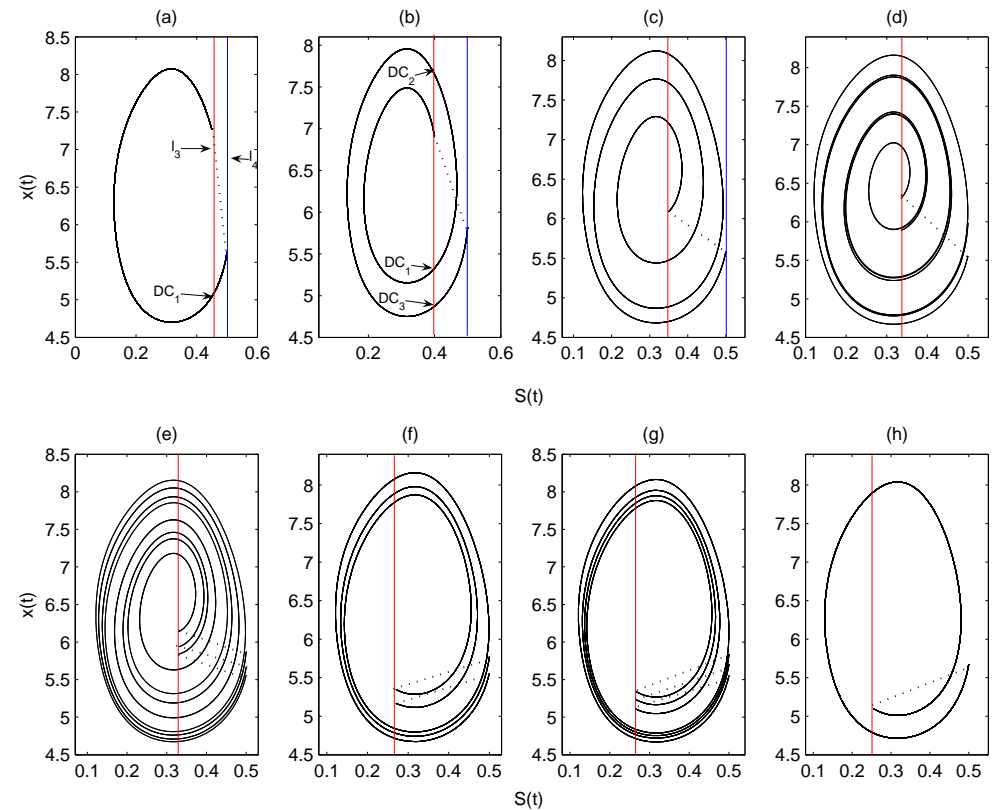

Fig. 7. Discontinuity points of Poincaré map under case $\left(S C_{3}\right)$. (a) $\delta=0.1$, order-1 periodic solution with one discontinuity point; (b) $\delta=0.2$, order-1 periodic solution with three discontinuity points; (c) $\delta=0.3$, order-1 periodic solution with six discontinuity points; (d) $\delta=0.33$ order-1 periodic solution with eight discontinuity points; (e) $\delta=0.34$, order-3 periodic solution with finite discontinuity points; (f) $\delta=0.46$, order- 2 periodic solution with six discontinuity points; (g) $\delta=0.47$, order-3 periodic solution with eight discontinuity points; (h) $\delta=0.49$, order-1 periodic solution with two discontinuity points. $D C_{i}$ denotes the discontinuity points $(i=1,2,3, \cdots)$. Other parameter values are fixed as: $D=0.1, S_{F}=1.5, a=0.1, b=8$, $\mu_{\max }=0.4, K_{S}=0.95, \sigma=0.3, \tau=0.5$ and $C_{T}=0.5$.

In order to solve these problems, two cases were considered. For case $\left(A_{1}\right)$, since any solution from the phase set experiences pulse actions, we first defined the Poincaré map and then investigated its complex properties. These enable us to show the existence of order $-k$ periodic solution of system (3). First of all, the threshold condition for the existence and stability of the microorganism eradication periodic solution was provided. Besides, the PRCC results suggest that microbial extinction is dominated by the control parameter $\delta$ and microbial growth is determined by the control paramter $\sigma$. When the microorganism eradication periodic solution is unstable, the transcritical bifurcation was studied which means an order1 periodic solution is generated for system (3). It indicates that the microorganism density is stabilized along a periodic solution. After that, by discussing the fixed points of the Poincare map, we investigated the existence of the order- $k(k \geq 1)$ periodic solution. The results reveal that for system $(3)$ there exist periodic solutions with any period.

For case $\left(A_{2}\right)$, since the solution initiating from the phase set may not experience pulses, the exact domains of the impulsive sets which ensure the occurrence of a pulse action need to be discussed, and all the possible cases are shown in Tab.4.1. To avoid repetition, we just focussed on the dynamics that are different from case $\left(A_{1}\right)$. Theorem 8 told us that system (3) may only exist with an order-1 periodic solution or order-2 periodic solution. Under conditions of Remark 3, multi-stable behaviors are observed, and it reveals that the concentrations of the substrate and microorganism populations may stabilize at different fixed levels. Once $E^{*}$ is unstable, many interesting results are found. The dynamics of system (3) are not only complex, but also sensitive to the control parameters (Fig.6). Meanwhile, the Poincaré map has a complicated shape with discontinuity points, and the number of discontinuity points first increases and then decreases as the control parameter $\delta$ increases.

Compared to the previous studies of chemostat models with state-dependent feedback control [Sun et al., 2011; Tian et al., 2010], the following aspects are highlights: (1) the exact domains of impulsive sets are discussed for all cases; (2) the complex properties of the Poincaré map have been studied, and 
the discontinuity points have been addressed; (3) the transcritical bifurcation near the microorganism eradication periodic solution has been investigated, and it indicates that an order- 1 periodic solution is generated for system (3); (4) the global stability of the order-1 periodic solution has been proved, and the conditions for the existence of an order -3 periodic solution are provided; (5) multi-stable behaviors are observed under certain conditions; (6) system (3) exhibits very rich and complex dynamics other than with the order -1 or order -2 periodic solutions. All these aspects enrich and improve the results of chemostat models.

In this paper, we assumed that impulsive state feedback control is an instantaneous process. However, the control strategy from implementation to reach its purpose requires a certain time. The essential character of this process is hysteresis, how to depict and further realize this hysteresis process is a challenge in the near future. It is hoped that such research, planned for the near future and to be reported elsewhere, will be useful for microbial cultivation.

\section{Acknowledgments}

This work was supported by the Chongqing Municipal Education Commission (KJ1600522), Basic and Advanced Research Project of Chongqing( cstc2017jcyjAX0131, cstc2016jcyjA0412), and National Natural Science Foundation of China (NSFC 11761031).

\section{References}

Bailey, J. \& Ollis, D. [1986] Biochemical Engineering Fundamentals, 2nd edition, McGraw-Hill, New York.

Bainov, D. \& Simeonov, P. [1993] Impulsive Differential Equations: Periodic Solutions and Applications, vol. 66, Longman.

Butler, G. \& Wolkowicz, G. [1985] "A mathematical model of the chemostat with a general class of functions describing nutrient uptake", SIAM J. Appl. Math. 45, 138-151.

Ciesielski, K. [2004] "On stability in impulsive dynamical systems", Bull. Pol. Acad. Sci. Math. 52, 81-91.

Ciesielski, K. [2004] "On time reparametrizations and isomorphisms of impulsive dynamical systems", Ann. Polon. Math. 84, 1-25.

De Leenheer, P. \& Smith, H. [2003] "Feedback control for chemostat model", J. Math. Biol. 46, 48-70.

Devaney, R. [1989] "An introduction to chaotic dynamical systems", Addison-Wesley, New York.

Guo, H. \& Chen, L. [2009] "Periodic solution of a chemostat model with Monod growth rate and impulsive state feedback control", J. Theor. Biol. 260, 502-509.

Huang, M., Li, J., Song, X. \& Guo, H. [2012] "Modeling impulsive injections of insulin: Towards artificial pancreas", SIAM J. Appl. Math. 72, 1524-1548.

Kasperski, A. \& Miskiewicz, T. [2008] "Optimization of pulsed feeding in a Bakers yeast process with dissolved oxygen concentration as a control parameter", Biochem. Eng. J. 40, 321-327.

Kaul, S. [1990] "On impulsive semi-dynamical systems", J. Math. Anal. Appl. 150, 120-128.

Krishnaiah, D. [2007] "Chemical and bioprocess engineering: a special issue of applied sciences", J. Appl. Sci. 7, 1989-1990.

Li, Z., Wang, T. \& Chen, L. [2009] "Periodic solution of a chemostat model with Beddington-DeAnglis uptake function and impulsive state feedback control", J. Theor. Biol. 261, 23-32.

Li, T. \& Yorke, J. [1975] "Period three implies chaos", Amer. Math. 82, 985-992.

Liu, X. \& Chen, L. [2003] "Complex dynamics of Holling type II Lotka-Volterra predator-prey system with impulsive perturbations on the predator", Chaos Soliton. Fract. 16, 311-320.

Lobry, J., Flandrois, J., Carret, G. \& Pavemonod's, A. [1992] "Bacterial growth model revisited", Bull. Math. Biol. 54, 117-122.

Meng, X. \& Chen, L. [2008] "Permanence and global stability in an impulsive LotkaCVolterra n-species competitive system with both discrete delays and continuous delays", Int. J. Biomath. 1, 179-196.

Menkel, F. \& Knights, A. [1995] [1995] "A biological approach on modelling a variable biomass yield", Process. Biochem. 30, 485-495.

Rasband, S. [1990] Chaotic Dynamics of Nonlinear Systems, J. Wiley and Sons, New York. 
Rehm, H. \& Reed, J. [1981] Biotechnology, Microbial Fundamentals, Verlag Chemie, Weinheim.

Schugerl, K. \& Bellgardt, K. [2000] Bioreaction Engineering: Modeling and Control, Springer-Verlag, Berlin. Simeonov, P. \& Bainov, D. [1988] "Orbital stability of periodic solutions of autonomous systems with impulsive effect", Internat. J. Systems. Sci. 19, 2561-2585.

Simkins, S. \& Alexander, M. [1984] "Models for mineralization kinetics with the variables of substrate concentration and population density", Appl. Environ. Microbiol. 47, 1299-1306.

Simkins, S. \& Alexander, M. [1985] "Nonlinear estimation of the parameters of Monod kinetics that best describe mineralization of several substrate concentrations by dissimilar bacterial densities", Appl. Environ. Microbiol. 50, 816-824.

Sun, S. \& Chen, L. [2007] "Dynamic behaviors of Monod type chemostat model with impulsive perturbation on the nutrient concentration", J. Math. Chem. 42, 837-847.

Sun, K., Kasperski, A., Tian, Y. \& Chen, L. [2011] "Modelling and optimization of a continuous stirred tank reactor with feedback control and pulse feeding", Chem. Eng. Process. 50, 675-686.

Sun, K., Tian,Y., Chen, L. \& Kasperski, A. [2010] "Nonlinear modelling of a synchronized chemostat with impulsive state feedback control", Math. Comput. Model. 52, 227-240.

Tang, B., Xiao, Y., Tang, S. \& Cheke, R. [2015] "A Feedback Control Model of comprehensive Therapy for Treating Immunogenic Tumours", Int. J. Bifurcat. Chaos. 81, 1575-1596.

Thierie, J. [2004] "Modeling threshold phenomena, metabolic pathways switches and signals in chemostatcultivated cells: the Crabtree effect in Saccharomyces cerevisiae", J. Theor. Biol. 226, 483-501.

Tian, Y., Sun, K., Kasperski, A. \& Chen, L. [2010] "Nonlinear modelling and qualitative analysis of a real chemostat with pulse feeding", Discrete Dyn. Nat. Soc. Art. 1, 179-186.

Yang, J., Tang, G. \& Tang, S. [2017] "Modelling the regulatory system of a chemostat model with a threshold window", Math. Comput. Simlat. 132, 220-235.

Zeng, G., Chen, L. \& Sun, L. [2006] "Existence of periodic solution of order one of planar impulsive autonomous system", J. Comput. Appl. Math. 186, 466-481.

Zhao, Y. \& Skogestad, S. [1997] "Comparison of various control configurations for continuous bioreactors", Ind. Eng. Chem. Res. 36, R697-R705. 